\title{
17 Diagnosehäufigkeit und Inanspruchnahme von Gesundheitsleistungen
}

\author{
Caroline Schmuker, Ghassan Beydoun und Christian Günster
}

C. Günster | |. Klauber | B.P. Robra | C. Schmuker | A. Schneider (Hrsg.) Versorgungs-Report Klima und Gesundheit. DOI 10.32745/9783954666270-17, (C MWV Medizinisch Wissenschaftliche Verlagsgesellschaft Berlin 2021

Der Beitrag berichtet für das Jahr 2018 die Häufigkeit von Erkrankungen und Behandlungen in Deutschland. Die Analysen basieren auf standardisierten Abrechnungsdaten von AOK-Versicherten. Dargestellt werden administrative Behandlungsprävalenzen nach den dreistelligen Diagnoseschlüsseln und den Diagnoseobergruppen des ICD-10. Zusätzlich werden in den vier ausgabenwirksamsten Leistungssektoren (stationäre Versorgung, ambulant-ärztliche Versorgung, Arzneimittel- und Heilmittelversorgung) Kennziffern zur Inanspruchnahme von Gesundheitsleistungen berichtet.

This article reports the frequency of diseases and treatments in Germany for 2018. Analyses are based on standardised administrative claims data of the Local Health Insurance Funds (AOK). The article presents administrative prevalence rates according to three character ICD-10 diagnosis codes and ICD-10 main groups. In addition, key figures on the utilisation of health services in the four most cost-intensive sectors of the health care system are reported separately: inpatient care, outpatient care, pharmaceutical and remedy care.

\subsection{Einführung}

Das Wissenschaftliche Institut der AOK (WIdO) stellt regelmäßig seit 2011 mit dem VersorgungsReport Kennzahlen zum Krankheitsgeschehen und zur Inanspruchnahme von medizinischen Leistungen zur Verfügung. Der aktuelle Beitrag stellt administrative Behandlungsprävalenzen nach den dreistelligen ICD-10-Diagnoseschlüsseln und nach übergreifenden Diagnoseobergruppen bezogen auf das Jahr 2018 dar. Die Diagnoseinformationen werden ergänzt um Hospitalisierungsraten, sodass die Bedeutung einer Erkrankung für die stationäre Versorgung transparent wird. Darüber hinaus nimmt der Versorgungs-Report die Versorgung der Versicherten innerhalb der vier Leistungsbereiche stationäre Versorgung, ambulante vertragsärztliche Versorgung, Arznei- und Heilmittelversorgung in den Blick. Alters-und geschlechtsspezifische Kennzahlen der Inanspruchnahme werden bundesweit sowie in regionaler Differenzierung (kartografisch) ausgewiesen. In Ergänzung zu diesem Kapitel bietet der Versorgungs-Report ta- 
bellarische Übersichten über die Behandlungshäufigkeiten von mehr als 1.500 dokumentierten Einzeldiagnosen bzw. 268 Diagnoseobergruppen sowie weitere Einzelauswertungen, die als elektronischer Anhang zu diesem Buchkapitel zur Verfügung gestellt werden ${ }^{1}$.

Darstellung und Analysen im VersorgungsReport sind personenbezogen, d.h. Leistungsoder Diagnoseinformationen aus verschiedenen Sektoren werden versichertenbezogenen (anonymisiert) zusammengeführt und ausgewertet. Erst der Personenbezug erlaubt die Schätzung von epidemiologischen Kennzahlen wie Prävalenzen und Inzidenzen. Fallbezogene Statistiken dagegen (z.B. die Krankenhausstatistik des Statistisches Bundesamtes) ermöglichen zwar Aussagen zur Zahl der Krankenhausfälle, lassen aber keinen Rückschluss auf die Zahl der Personen mit einer Erkrankung oder die Häufigkeit der Krankenhausaufenthalte eines Patienten zu.

Die vorliegenden Auswertungen basieren auf den Routinedaten von AOK-Versicherten im Jahr 2018, die mehr als ein Drittel der gesetzlichen Krankenversicherung (GKV) und mehr als $30 \%$ der deutschen Bevölkerung repräsentieren $^{2}$. Kennzahlen in diesem Beitrag sind hinsichtlich Alter und Geschlecht standardisiert und auf die deutsche Wohnbevölkerung des Jahres 2018 hochgerechnet.

Der Beitrag gliedert sich in die Beschreibung der Datengrundlage (s. Kap. 17.2) Methoden (s. Kap. 17.3) und Limitationen bei der Nutzung der AOK-Versichertendaten (s. Kap. 17.4) sowie die Darstellung der Kennzahlen zur Behandlungshäufigkeit (s. Kap. 17.5) und Inanspruchnahme von Gesundheitsleistungen (s. Kap. 17.6) im Jahr 2018.

1 Auffindbar im Open Access-Portal der Medizinisch Wissenschaftlichen Verlagsgesellschaft: https://www.mwv-open.de/ site/books/e/10.32745/9783954666270/

2 Laut Mitgliederstatistik des Bundesministeriums für Gesundheit gab es im Jahresdurchschnitt 2018 26,5 Mio. AOK-Versicherte und 72,8 Mio. GKV-Versicherte (jeweils inkl. mitversicherter Angehöriger). Stand der deutschen Wohnbevölkerung am 31.12.2018 war laut Statistischem Bundesamt 83,0 Mio.

\subsection{Datengrundlage}

\subsubsection{Abrechnungsdaten}

Datengrundlage für diesen Beitrag sind die bundesweiten anonymisierten Abrechnungsdaten aller AOK-Versicherten mit mindestens einem Versichertentag im Auswertungsjahr. Für die präsentierten Querschnittsanalysen des Jahres 2018 liegen Angaben von 27,8 Mio. Versicherten vor (s. Abb. 1). Die im Folgenden dargestellten Kennzahlen werden jeweils nach Geschlecht, sowie differenziert für die Altersgruppen Kinder und Jugendliche (1 bis 17 Jahre), mittlere Erwachsene (18 bis 59 Jahre) und ältere Erwachsen (6o Jahre und älter) aufbereitet.

Für diesen Beitrag wurden die folgenden Abrechnungs- und Stammdaten versichertenbezogen (anonymisiert) zusammengeführt und ausgewertet:

- Versichertenstammdaten (nach $\mathbb{S}$ 288, SGB V)

- ambulante vertragsärztliche Versorgung

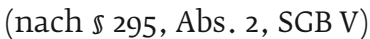

- Arzneimittelabrechnung (nach $\$ 300$, Abs. 1, SGB V)

n stationäre Versorgung (nach $\mathbb{3} 301$, Abs. 1, SGB V)

- Heilmittelversorgung (nach $\mathbb{3} 302$, Abs. 1, SGB V)

Die Daten geben Auskunft über die in Deutschland behandelten Erkrankungen, so wie sie von Ärzten dokumentiert werden, und darüber, welche therapeutischen und diagnostischen Leistungen in der Behandlung erbracht wurden. Dabei können verschiedene Institutionen und Personen (Kliniken und niedergelassene Ärzte) beteiligt gewesen sein .

Die folgenden Abschnitte beschreiben den sozialrechtlichen Rahmen der Leistungsdatenübermittlung und präzisieren die Kriterien, die für die Datenselektion in diesem Beitrag angewendet wurden. 


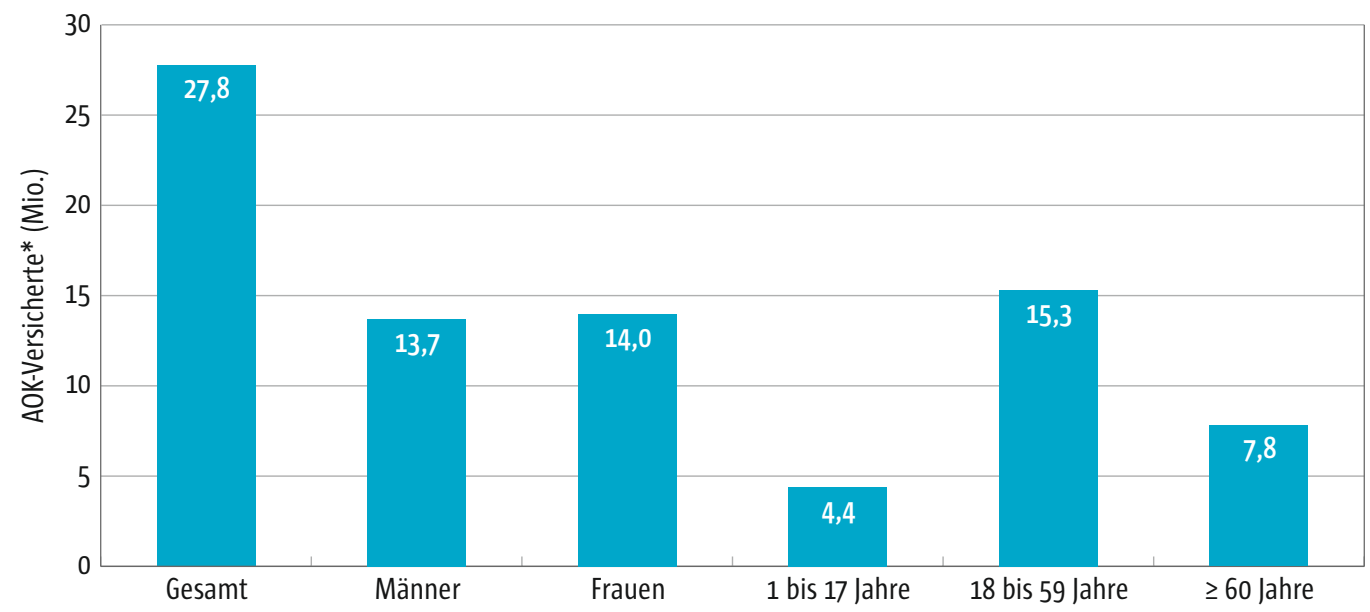

* Basierend auf allen Personen, die im Jahr 2018 mindestens einen Tag bei der AOK versichert waren. Umfasst daher mehr Personen als der Jahresdurchschnitt 2018 der Mitgliederstatistik des BMG (KM1/13). Zielpopulation für die folgenden Auswertungen.

Abb. 1 AOK-Versicherte nach Geschlecht und Altersgruppen im Jahr 2018 (in Mio.)

\section{Arzneimittelversorgung}

Gemäß $\mathbb{3} 300$ SGB V werden Daten zu allen verschreibungspflichtigen Fertigarzneimitteln und Nicht-Fertigarzneimitteln übermittelt, die von einem niedergelassenen Vertragsarzt auf Rezepten zulasten der GKV verordnet und über eine öffentliche Apotheke abgerechnet wurden. Dabei werden auch Angaben zum Apothekenverkaufspreis, zum Verordnungs- und Abgabedatum sowie zum verordnenden Arzt dokumentiert. Das Verordnungsdatum bestimmt die Zuordnung der Leistung zum Berichtszeitraum. Ausgabenschätzungen für Arzneiverordnungen zulasten der GKV basieren auf dem jeweiligen Bruttoumsatz inklusive Zuzahlung durch den Versicherten (Apothekenverkaufspreis). Fertigarzneimittel lassen sich durch die sogenannte Pharmazentralnummer eindeutig einem Handelsnamen, dem Hersteller, der Wirkstoffstärke sowie der Packungsgröße zuordnen. Auf Basis der Pharmazentralnummer werden im WIdO die Zuordnungen von Fertigarzneimitteln zu den jeweiligen Wirkstoffgruppen vorgenommen (vgl. Kap. 17.2.2). Für die Analysen in diesem Beitrag wurden im Wesent- lichen alle Verordnungen von Fertigarzneimitteln, Rezepturen, Diagnostika und Substitutionstherapie-Präparate berücksichtigt.

\section{Stationäre Versorgung}

Im Rahmen der stationären Versorgung von GKV-Versicherten übermitteln die Kliniken je Behandlungsfall Angaben zum Versicherten, zum Aufnahme- und Entlassungsdatum, Diagnosedaten sowie den Rechnungsbetrag. Die Entlassungsdiagnosen - obligate Hauptdiagnose und fakultative Nebendiagnose(n) - sind im Rahmen der Krankenhausabrechnung rechnungsbegründend und werden daher systematisch erfasst. Für die Analysen wurden alle abgeschlossenen voll- und teilstationären Aufenthalte ausgewertet. Leistungen wurden gemäß Entlassungsdatum dem Behandlungsjahr zugeordnet. Zur Bestimmung von Behandlungsprävalenzen wurde auf die Hauptund Nebendiagnose der stationären Behandlung zurückgegriffen. Der primäre Behandlungsanlass wurde über die Hauptdiagnose erfasst. 


\section{Ambulante Versorgung}

Grundlage sind die von einem ambulant tätigen Vertragsarzt durchgeführten Leistungen des GKV-Leistungsbereichs. Diese Leistungen werden, sofern es sich um kollektivvertragliche Leistungen handelt, einmal pro Quartal über eine der 17 regional zuständigen Kassenärztlichen Vereinigungen abgerechnet und die Daten anschließend an die Krankenkassen weitergeleitet. Als ein Behandlungsfall gilt die Konsultation eines Versicherten bei einem Vertragsarzt in einem Quartal; dabei ist die Anzahl der Praxisbesuche im Quartal unerheblich. Eine Person erzeugt mehr als einen Behandlungsfall pro Quartal, wenn sie im selben Quartal mehrere Ärzte aufsucht. Zu jedem Abrechnungsfall werden quartalsweise die Behandlungsdiagnosen mit Angabe der Diagnosesicherheit (gesicherte Diagnose, ausgeschlossene Diagnose, Verdachtsdiagnose, symptomloser Zustand) kodiert. Gemäß $\mathbb{2} 295$ SGB V sind in beiden Fällen die amtlichen und aktuell gültigen Fassungen des DIMDI zu nutzen (vgl. Kap. 17.2.2). Zur Ermittlung der hier dargestellten Behandlungsprävalenzen werden ausschließlich gesicherte Diagnosen herangezogen. Es werden Behandlungsdiagnosen aus kollektivvertraglicher und selektivvertraglicher Versorgung berücksichtigt.

\section{Neue Zuordnung der Behandlungsfälle zu EBM-Facharztgruppen}

Mit der vorliegenden Ausgabe des VersorgungsReportes wird das Verfahren zur Auswertung der fachärztlichen Inanspruchnahme umgestellt. Im bisherigen Verfahren wurden Behandlungsfälle über die Betriebsstättennummer (BSNR) des Vertragsarztsitzes einer Facharztgruppe zugeordnet. Die BSNR ermöglicht es, über eine Schlüsseltabelle (nach Richtlinie der Kassenärztlichen Bundesvereinigung [KBV]) die Facharztgruppe des abrechnenden Arztes eindeutig zu ermitteln. Arztpraxen (Betriebsstätten) in denen mehrere Ärzte verschiedener
Facharztrichtungen tätig sind (z.B. als Gemeinschaftspraxis organisierte Facharztpraxen) wurden in der bisherigen Darstellung der Kategorie „fachgruppenübergreifende Facharztpraxen“ zugeteilt. Allerdings nimmt der Anteil der Personen, die in fachübergreifenden Facharztpraxen behandelt werden, stetig zu (2010: 47,3\%; 2016: 52,1\%), was zu einer zunehmenden Unterschätzung der Behandlungsraten bei einzelnen Facharztgruppen führt. Das betrifft insbesondere Facharztgruppen, die häufig in Gemeinschaftspraxen tätig sind, darunter z.B. die fachärztlichen Bereiche Orthopädie und Chirurgie.

Das neue Verfahren zur Bestimmung der Facharztgruppe setzt auf den Grund- und Versichertenpauschalen der jeweiligen Fachärzte gemäß dem EBM-Katalog auf. Die EBM-Facharztgruppen werden für die Darstellung im Versorgungs-Report zu 16 Facharztgruppen zusammengefasst (EBM-Fachgruppenzuordnung s. elektronischer Anhang). Wie zuvor werden Leistungen aus Kollektiv- und selektivvertraglicher Versorgung berücksichtigt. Bei der neuen Auswertung nach EBM-Facharztgruppen sind einige Besonderheiten zu berücksichtigen:

- Die Kategorie „Hausarzt“ ersetzt die Facharztgruppe der „Allgemeinmediziner“. Es werden alle Behandlungsfälle gezählt, die zur Abrechnung einer hausärztlichen Grundpauschale geführt haben. Insofern können auch andere Facharztgruppen (z.B. Kardiologen) an der Versorgung beteiligt gewesen sein, sofern sie jeweils eine Zulassung oder Ermächtigung zur Teilnahme an der hausärztlichen Versorgung hatten.

- Die Kategorie „Notfall“ ist ein gesonderter vertragsärztlicher Versorgungsbereich, an dem sich grundsätzlich alle EBM-Facharztgruppen über den kassenärztlichen Bereitschaftsdienst sowie die Notfallambulanzen der Krankenhäuser beteiligen.

- In der Kategorie „Weitere vertragsärztliche Leistungen ohne Versicherten- bzw. Grundpauschale "werden Behandlungsfälle gezählt, für die keine Versicherten- bzw. Grundpauschale und keine ambulanten Notfallleistungen nach dem 
EBM-Kapitel 1.2 abgerechnet wurden. Hierbei handelt es sich oftmals um Leistungen ohne Versichertenkontakt, darunter bestimmte Laborleistungen oder Sachkosten (z.B. Arztbriefe).

- In der Kategorie „Fälle mit mehreren Grundpauschalen“ (s. Abb. 10) werden Behandlungsfälle mit mehr als einer Grund- bzw. Versichertenpauschale gezählt. Dabei handelt es sich häufig um labormedizinische Leistungen in Verbindung mit einer gynäkologischen Konsultation. Diese Behandlungsfälle werden in der Darstellung gesondert ausgewiesen.

\section{Heilmittelversorgung}

Basis sind Heilmittelleistungen - also Physiotherapie, Ergotherapie, Podologie und Sprachtherapie -, die von einem niedergelassenen Vertragsarzt zulasten der GKV verordnet und von einem zur Heilmittelversorgung zugelassenen Leistungsanbieter erbracht werden. Indikationen, die zur Verordnung eines Heilmittels führen, werden nach dem im jeweiligen Auswertungsjahr gültigen Heilmittelkatalog klassifiziert. Die erbrachten Leistungen lassen sich über eine fünfstellige bundeseinheitliche Heilmittelpositionsnummer eindeutig zuordnen. Bei der Abrechnung erhalten die Krankenkassen außerdem die auf der Heilmittelverordnung dokumentierten Angaben zum Versicherten (Alter, Geschlecht, Wohnort), zum verordnenden Arzt, zum Verordnungsdatum sowie zum Leistungserbringer. Bei Zählung der Leistungen wurden nur therapeutische Leistungen berücksichtigt (ohne Zusatzleistungen).

\subsubsection{Klassifikationen}

\section{Klassifikation von Erkrankungen}

Die für Deutschland modifizierte Internationale statistische Klassifikation der Krankheiten (ICD10-GM) ist die amtliche Klassifikation für Diag- nosen in der ambulanten und stationären Versorgung in Deutschland. Für die Analysen des Beitrags wird die Ausgabe der ICD-10-GM für das Jahr 2018 genutzt (DIMDI 2018a). Die vorliegenden Analysen basieren auf den ICD-Schlüsselnummern der Haupt- und Nebendiagnosen stationärer Behandlungen sowie den gesicherten Diagnosen aus der ambulanten vertragsärztlichen Versorgung. Im vorliegenden Kapitel erfolgte die Auswertung der Behandlungshäufigkeiten auf der Basis von dreistelligen Einzeldiagnosen (ICD-10-Dreisteller). In Ergänzung hierzu werden Behandlungshäufigkeiten auf Ebene der ICD-10-Diagnoseobergruppen im elektronischen Anhang zur Verfügung gestellt. Abweichend vom ICD-10-Katalog werden in diesem Beitrag Obergruppen weiter unterteilt, um zwischen akuten Zuständen und i.d.R. nicht behandlungsbedürftigen Erkrankungen zu differenzieren. Auf diese Weise sind 268 Obergruppen entstanden - die Modifikationen sind in der Auswertung mit einem Sternchen $\left(^{*}\right)$ am ICDCode gekennzeichnet

\section{Klassifikation von Arzneimittelwirkstoffen}

Wirkstoffe von Fertigarzneimitteln werden auf der Basis der eindeutigen Pharmazentralnummer nach der Anatomisch-Therapeutischen Klassifikation (ATC) verschlüsselt. Für diesen Beitrag wird der ATC-Index des GKV-Arzneimittelindex des jeweiligen Auswertungsjahres (hier 2018) verwendet, der Spezifizierungen für den deutschen Arzneimittelmarkt enthält und in dem auch die definierten Tagesdosen (DDD) festgelegt sind (DIMDI 2018b). Das WIdO passt diese Systematik kontinuierlich an die Besonderheiten der Versorgungssituation in Deutschland an (Fricke et al. 2018)

\section{Raumordnungsregionen}

Als Bezugsrahmen für die großräumigen Analysen von Diagnose- und Erkrankungshäufig- 
keiten in diesem Beitrag wurden die 96 Raumordnungsregionen des Bundesinstituts für Bau-, Stadt- und Raumforschung (BBSR 2017) verwendet. Die Zuordnung erfolgt auf Basis der in den Daten der Mitgliederbestandsführung gespeicherten Postleitzahl des Versichertenwohnortes. Die Zuordnung des Versichertenwohnortes zu einem Bundesland geschieht über den Kreis-Cemeindeschlüssel, der jährlich von der Post zur Verfügung gestellt wird. Zu beachten ist, dass sich die empirisch festgelegten Raumordnungsregionen nicht zwangsläufig mit den amtlich festgelegten Regionalgrenzen von Gemeinden, Kreisen oder Bundesländern decken. Bei Stadtstaaten wie beispielsweise Bremen können die für das Bundesland dokumentierten Behandlungshäufigkeiten daher von den Behandlungshäufigkeiten der Raumordnungsregion Bremen abweichen.

\subsection{Methoden}

\subsubsection{Alters- und Geschlechts- standardisierung}

Die Alters- und Geschlechtsstruktur der AOKVersicherten unterscheidet sich teilweise von der der bundesdeutschen Wohnbevölkerung. So liegt der Anteil der Frauen im Alter von 35 bis 65 Jahren bei AOK-Versicherten unter dem Bundesdurchschnitt, während Frauen ab einem Alter von 75 Jahren in der AOK überproportional häufig vertreten sind (s. Abb. 2). Männer sind vor allem im jüngeren Erwachsenenalter zwischen 18 und 35 Jahren in der AOK überrepräsentiert, im höheren Erwachsenenalter (zwischen 45 und 75 Jahren) hingegen liegt der Anteil der Männer (vergleichbar zu den Frauen) unter dem Bundesdurchschnitt, wenngleich die Differenz zur deutschen Wohnbevölkerung bei ihnen geringer ausfällt als bei den Frauen. Zudem weist die AOK im Jahr 2018 einen überdurchschnittlichen Anteil an hochbetagten Menschen (insbesondere Frauen über 8o Jahre) auf. Da diese Merkmale einen Einfluss auf Morbidität und In- anspruchnahme von Gesundheitsleistungen haben, wurde in diesem Beitrag zur Berechnung der verschiedenen Kennzahlen eine direkte Alters- und Geschlechtsstandardisierung vorgenommen (Kreienbrock et al. 2012). Dabei wurden für die AOK-Versicherten die entsprechenden Kennzahlen in Geschlechts- und Altersklassen berechnet und mit der Geschlechts- und Alterszusammensetzung der deutschen Wohnbevölkerung gewichtet. Es wurden die in Abbildung 2 gezeigten Altersklassen genutzt. Bei alters- und geschlechtsübergreifenden Darstellungen wurden die derartig gewichteten Kennzahlen entsprechend aufsummiert. Bei den regionalisierten Darstellungen wurde die Vergleichbarkeit der einzelnen Regionen hergestellt, indem auch hier die Alters- und Geschlechtsstruktur in ganz Deutschland zugrunde gelegt und auf die deutsche Wohnbevölkerung standardisiert wurde. Unterschiede in der (regionalen) Inanspruchnahme von medizinischen Leistungen sind somit nicht auf demografische Unterschiede zurückzuführen.

\subsubsection{Kennzahlen für Behandlungs- häufigkeiten und die Inanspruch- nahme von Gesundheitsleistungen}

\section{Bestimmung der administrativen Behandlungs- prävalenzen}

Dieser Beitrag weist Behandlungsprävalenzen für die häufigsten Erkrankungen bzw. Behandlungsanlässe für das Jahr 2018 aus. Die Jahresprävalenz einer Erkrankung wurde definiert als die Anzahl aller Personen mit der Zieldiagnose (Analysepopulation) bezogen auf alle Versicherten mit mindestens einem Versichertentag im Jahr 2018. Die Daten dafür beruhen auf den stationär gestellten oder - wenn keine Krankenhausbehandlung vorlag - auf ambulant dokumentierten gesicherten Diagnosen. Sämtliche ausgewiesenen Prävalenzen sind daher als dokumentierte Behandlungsprävalenz bzw. administrative Prävalenz zu interpretieren. Allerdings beziehen 


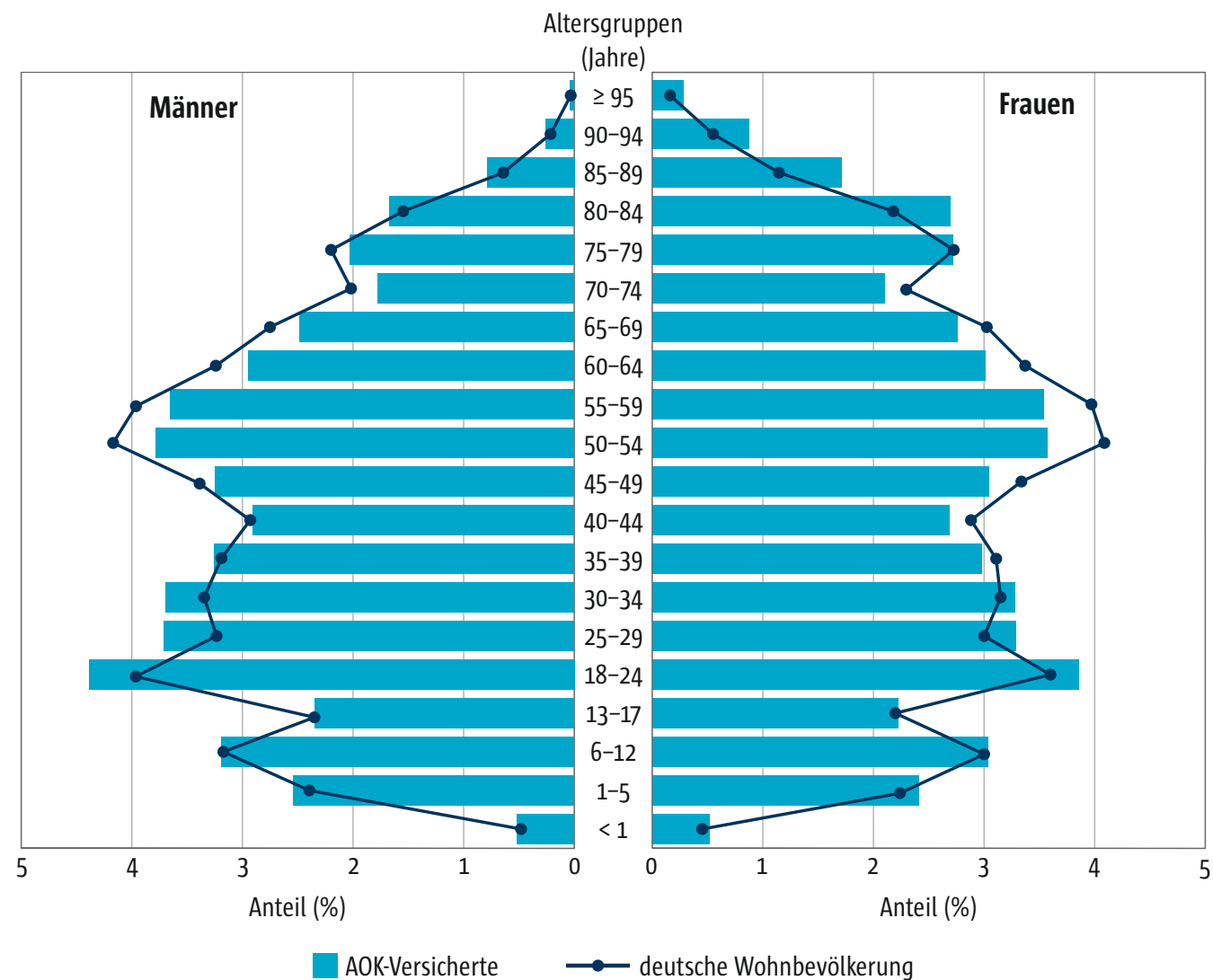

Abb. 2 Alters- und Geschlechtsverteilung der deutschen Wohnbevölkerung und der AOK-Versicherten im Jahr 2018

sich die dargestellten Behandlungsprävalenzen nicht nur auf Erkrankungen, sondern auch auf andere Behandlungsanlässe, z.B. Faktoren, die den Gesundheitszustand beeinflussen und zur Inanspruchnahme des Gesundheitswesens führen (ICD-1o Zoo-Z99). Diese Kodierung wird beispielsweise für Früherkennungsuntersuchungen, Impfungen oder Ceburten verwendet. Derartige Maßnahmen können auch gesunde Personen in Anspruch nehmen. Sie spiegeln nicht unbedingt die Morbidität wider, stellen aber doch eine Inanspruchnahme des Cesundheitswesens dar. Bei seltenen Diagnosegruppen wurde die Prävalenz aufgrund von Rundungen als o,o (d.h. $<$ o, 05) ausgewiesen. Gruppen mit weniger als hochgerechnet 1.00o Personen sind in den Tabellen nicht dargestellt.

\section{Validierung der dokumentierten Diagnosen}

Stationäre Diagnosenennungen werden als verlässlich bewertet, da die Kodierung von Krankenhausdiagnosen mehreren, stetig geschärften Prüfmechanismen unterliegt (s. Kap. 17.4). Aus diesem Grund werden als Analysepopulation alle Personen aufgegriffen, die einen Krankenhausaufenthalt mit der jeweiligen Zieldiagnose (als Haupt- oder Nebendiagnose) im Berichtsjahr oder -zeitraum aufweisen. Die von niedergelassenen Medizinern in ambulanter Praxis dokumentierten Diagnosen, die ohne konsentierte Kodierrichtlinien erstellt werden, bedürfen einer genaueren Prüfung. Bei ausschließlich ambulant behandelten Personen muss im Falle einer chronischen Erkrankung 
die betreffende Diagnose daher in mindestens zwei von vier Quartalen (auch M2Q-Kriterium genannt) dokumentiert sein. In Abhängigkeit von der jeweiligen Zielerkrankung gelten allerdings unterschiedliche Bezugszeiträume:

Bei Erkrankungen mit kontinuierlichem Krankheitsverlauf und Behandlungsbedarf (z.B. Herzinsuffizienz) ist der Bezugszeitraum das Kalenderjahr. Die Diagnosevalidierung erfolgt innerhalb der vier Quartale des Berichtsjahres.

\subsection{Limitationen und Validität von AOK-Routinedaten}

Die Abrechnungsdaten von mehr als 27,8 Mio. AOK-Versicherten geben die Chance, sektorenübergreifend Langzeitverläufe von großen Populationen ohne regionale Eingrenzung und ohne Beschränkung auf einen einzelnen Leistungssektor zu analysieren. Dennoch sind folgende Limitationen bei der Interpretation der dargestellten Ergebnisse zu berücksichtigen.

\section{Validität der dokumentierten Diagnose- informationen}

Die von ärztlichen Leistungserbringern dokumentierte Behandlungsmorbidität kann aus mehreren Gründen von der wahren Prävalenz einer Erkrankung abweichen:

- Über Diagnosenennungen können bestenfalls therapierte Erkrankte ermittelt werden. Erkrankte ohne Arztkontakt bleiben unerkannt.

- Diagnosen können fehlerhaft nach der ICDSystematik verschlüsselt werden.

- Bei multimorbiden Patienten können bei konkurrierenden Diagnosen tatsächlich vorliegende Erkrankungen ungenannt bleiben, wenn nur die vergleichsweise ,höherwertige“ Diagnose aufgezeichnet wird.

- Aus Gründen praxisinterner Abläufe können im ambulanten Bereich möglicherweise
Diagnosen ungewollt über mehrere Abrechnungsquartale hinweg fortgeführt werden, obwohl eine Erkrankung nicht mehr besteht.

- Diagnosestellungen haben oftmals eine legitimatorische Funktion in der jeweiligen Vergütungssystematik. Sie bezeichnen primär den Beratungs- und Behandlungsanlass und begründen das weitere ärztliche Handeln. Insofern spiegeln die dokumentierten Diagnosen nur bedingt die Morbidität wider. Mit der Einführung von diagnoseorientierten Fallpauschalen zur Vergütung von Krankenhausleistungen im Jahr 2003 wurde die Diagnosekodierung bestimmend für die Erlössituation der Krankenhäuser. Die Diagnosekodierung wird seitdem geregelt durch die Deutschen Kodierrichtlinien und ist Gegenstand der Abrechnungsprüfung der Krankenkassen und ihrer medizinischen Dienste. Die Kodierqualität gilt im stationären Bereich daher als verlässlich.

- Für den ambulanten Bereich liegen bislang keine entsprechenden Kodierrichtlinien vor. Die Dokumentation von Diagnosen durch hausärztlich tätige Ärzte kann (theoretisch) relativ unscharf sein, denn für Hausärzte ist das endstellige Kodieren nicht obligatorisch. Eine aktuelle empirische Untersuchung zeigt jedoch, dass die Kodierqualität unter Hausärzten durchaus gut ist. Demnach verwenden Hausärzte mittlerweile nicht nur eine Vielzahl sehr unterschiedlicher ICD10-Diagnosen, sondern nutzen in der Praxisrealität auch die Möglichkeit zum endstelligen Kodieren (Carnarius et al. 2018).

Auch wenn die Verlässlichkeit der Diagnosequalität zunimmt, sollten Diagnosen immer unter Hinzuziehung weiterer Merkmale wie Diagnoseherkunft (stationär oder ambulant), Dokumentationsdauer, Medikation erkrankungsspezifischer Wirkstoffe oder - je nach Fragestellung - weiterer Merkmale validiert werden (Hartmann et al. 2016; Schubert et al. 2010). 


\section{Operationalisierung von Krankheiten}

Bei der Nutzung von routinedatenbasierten Prävalenzangaben ist neben der Diagnosevalidierung auch das methodische Vorgehen zur Krankheitsdefinition von Bedeutung. Mit der Krankheits- bzw. Falldefinition wird festgelegt, welche Kriterien zur Bestimmung einer Erkrankung in Routinedaten herangezogen werden sollen. Die in diesem Beitrag dargestellten Prävalenzangaben basieren auf der ärztlichen Inanspruchnahme mit entsprechend validierter ICD-Kodierung (Behandlungsprävalenz). Dieses Vorgehen erlaubt Angaben zu Prävalenzen und Hospitalisierungsraten für die 1.500 häufigsten Erkrankungsgruppen und ermöglicht somit eine umfassende Einschätzung der epidemiologischen Bedeutung von Erkrankungen in Deutschland. Zur Abbildung von Krankheiten in Sekundärdaten sind jedoch auch komplexere Krankheitsdefinitionen möglich. Je nach Kontext der Forschungsfrage werden Krankheiten über verschiedene ICD-Schlüssel zusammengefasst oder mit weiteren Kriterien der Leistungsinanspruchnahme (z.B. Arzneimittelverordnungen oder spezifischen EBMLeistungen) validiert. Ein Beispiel hierfür sind die spezifischen Falldefinitionen des WIdO, die zur Berechnung von Krankheitshäufigkeiten in Deutschland im Rahmen des vom Innovationsfonds geförderten Projekts „BURDEN 2020“ verwendet werden (Breitkreuz et al. 2021). Hierfür wurden Krankheitsdefinitionen für 18 ausgewählte Erkrankungen und Schweregrade auf Basis von Routinedaten operationalisiert. Das entsprechende methodische Vorgehen mit den angewendeten Krankheitsdefinitionen, dem Prävalenzkonzept, dem alters-, geschlechtsund morbiditätsadjustierenden Hochrechnungsverfahren wie auch die ermittelten Prävalenzen für alle Einwohner Deutschlands sind unter www.krankheitslage-deutschland.de zugänglich.

\section{Repräsentativität der Daten}

Die Übertragbarkeit der in diesem Beitrag ausgewiesenen Kennzahlen auf die deutsche Wohnbevölkerung kann trotz der vorgenommenen Alters- und Geschlechtsstandardisierung eingeschränkt sein. Denn neben Alter und Geschlecht gibt es weitere Einflussfaktoren, z.B. soziodemografische Merkmale einer Person, die die Morbidität und Inanspruchnahme von Gesundheitsleistungen beeinflussen. Da sich die AOK-Versicherten möglicherweise in soziodemografischen Merkmalen von denen der deutschen Wohnbevölkerung unterscheiden, ist trotz Standardisierung bei der Hochrechnung auf die deutsche Wohnbevölkerung eine Über- oder Unterschätzung der betrachteten Maßzahlen denkbar (Hoffmann u. Icks 2012). Die Daten einer einzelnen Krankenkasse können daher keinen Anspruch auf vollständige Repräsentativität erheben (Jaunzeme et al. 2013). Ferner ist bei der Interpretation der vorliegenden Daten zu berücksichtigen, dass die AOK in den letzten Jahren einen starken Versichertenzuwachs erfahren hat. Laut der Mitgliederstatistik des Bundesministeriums für Gesundheit $(\mathrm{KM} / 13)$ ist die Zahl der AOK-Versicherten im Jahresdurchschnitt 2015 von 24,5 Mio. auf 26,5 Mio. im Jahr 2018 um rund 2 Mio. Versicherte gestiegen (BMG 2019). Da der hohe Versichertenzuwachs möglicherweise auch Auswirkungen auf die Morbiditätsstruktur der AOK hat, wird auch in der diesjährigen Ausgabe des Versorgungs-Reportes auf Vorjahresvergleiche verzichtet.

\section{Einschränkung auf Leistungen der gesetzlichen Krankenversicherung}

Die verwendete Datenbasis bildet den medizinischen Leistungsbedarf in den dargestellten Leistungsbereichen fast vollständig ab, soweit die Leistungen im GKV-Leistungskatalog enthalten sind. Grundsätzlich sind bei GKV-Routinedaten folgende Einschränkungen zu beachten: 
- In den Routinedaten fehlen individuelle Gesundheitsleistungen (IGel), deren Umfang in den letzten Jahren kontinuierlich gestiegen ist (Zok 2015).

- In den Arzneimittelverordnungsdaten sind nur die von niedergelassenen Vertragsärzten verordneten, in öffentlichen Apotheken eingelösten und mit den gesetzlichen Krankenkassen abgerechneten Arzneimittelrezepte berücksichtigt. Wenn Patienten Medikamente in der Apotheke selbst bezahlen, dann liegt der Krankenkasse darüber keine Information vor, obwohl die Leistung selbst im GKV-Leistungskatalog enthalten sein kann. Dies ist relativ häufig bei sogenannten OTCPräparaten (Over the Counter) der Fall, wenn - wie bei der Acetylsalicylsäure - der Packungspreis unterhalb des Zuzahlungsbetrags liegt; es ist seltener der Fall, wenn ein Versicherter einen Selbstbehalt-Tarif seiner Krankenkasse gewählt hat und aufgrund dessen eine Verordnung selbst bezahlt.

- Es liegen keine Informationen darüber vor, welche Arzneimittel im Rahmen stationärer Aufenthalte verabreicht wurden.

\subsection{Administrative Behandlungs- prävalenzen}

Tabelle 1 zeigt die 30 häufigsten dokumentierten Einzeldiagnosen bzw. Behandlungsanlässe in der Gesamtbevölkerung im Jahr 2018 differenziert nach Altersgruppen und Geschlecht. Eine erweiterte Übersicht über im Jahr 2018 dokumentierte Einzeldiagnosen (mit mehr als 1.00o Betroffenen) steht im elektronischen Anhang zur Verfügung.

Tabelle 1: Die Bedeutung der Tabellenspalten im Einzelnen

- Rang: Rangposition in der „Hitliste“ der häufigsten Erkrankungen

- ICD-10: dreistellige ICD-Schlüsselnummer nach ICD10-GM
Diagnose bzw. Behandlungsanlass: Klartextbeschreibung der ICD-Schlüsselnummer

- Prävalenz gesamt: Häufigkeit der Diagnose im Jahr 2018 insgesamt in der Bevölkerung. Anteil aller mit der Diagnose behandelten Personen an der Gesamtbevölkerung.

- Prävalenz männlich: Diagnosehäufigkeit in der männlichen Bevölkerung. Anteil der erkrankten männlichen Personen an allen männlichen Personen.

घ Prävalenz weiblich: Diagnosehäufigkeit in der weiblichen Bevölkerung. Anteil der erkrankten weiblichen Personen an allen weiblichen Personen.

- Prävalenz 1-17 Jahre: Diagnosehäufigkeit bei Kindern und Jugendlichen unter 18 Jahren. Neugeborene bis unter 1 Jahr werden aufgrund der besonderen Situation bei der Versorgung von Säuglingen/Frühgeborenen nicht berücksichtigt.

- Prävalenz 18-59 Jahre: Diagnosehäufigkeit bei Erwachsenen jüngeren und mittleren Alters von 18 bis unter 60 lahren

- Prävalenz 60 und mehr Jahre: Diagnosehäufigkeit bei älteren Erwachsenen ab 60 Jahren

- Hospitalisierungsrate allgemein: Anteil der Personen, die im Jahr 2018 in stationärer Behandlung waren. Dabei werden alle Krankenhausaufenthalte gezählt unabhängig von der betrachteten Diagnose.

- Hospitalisierungsrate mit dieser Hauptdiagnose: Anteil der Personen, die im Auswertungsjahr mit dieser Hauptdiagnose in stationärer Behandlung waren (d.h. diese Diagnose stellt den stationären Behandlungsanlass dar).

Die Diagnosen Rückenschmerzen (ICD-10 M54) und die essentielle (primäre) Hypertonie (ICD-10 I10) stellten mit einer Behandlungsprävalenz von $26,8 \%$ bzw. $26,6 \%$ die häufigsten dokumentierten Einzeldiagnosen der deutschen Wohnbevölkerung dar. Damit stehen beide Diagnosen - wie auch in den Vorjahren - unverändert an der Spitze der häufigsten Behandlungsanlässe (Gerste et al. 2014, 2016; Schmuker et al. 2019). In der Rangliste folgen akute Infektionen der oberen Atemwege (ICD-10 Jo6), von denen 22,0\% der Gesamtbevölkerung betroffen waren. Ein Viertel der Gesamtbevölkerung mit essentieller (primärer) Hypertonie (ICD-10 I10) war im Jahr 
Tab. 1 Prävalenzen und Hospitalisierungsquoten für die 30 häufigsten Behandlungsdiagnosen nach ICD-Dreistellern (2018)

\begin{tabular}{|c|c|c|c|c|c|c|c|c|c|c|}
\hline \multirow[b]{2}{*}{ Rang } & \multirow[b]{2}{*}{$\begin{array}{l}\text { ICD- } \\
10\end{array}$} & \multicolumn{5}{|c|}{ Prävalenz (in \%) } & \multirow[b]{2}{*}{$\begin{array}{l}18-59 \\
\text { Jahre }\end{array}$} & \multirow[b]{2}{*}{$\begin{array}{l}60 \text { und } \\
\text { mehr Jahre }\end{array}$} & \multicolumn{2}{|c|}{$\begin{array}{l}\text { Hospitalisierungsrate } \\
\text { gesamt (in \%) }\end{array}$} \\
\hline & & Diagnose bzw. Behandlungsanlass & gesamt & $\begin{array}{l}\text { männ- } \\
\text { lich }\end{array}$ & $\begin{array}{l}\text { weib- } \\
\text { lich }\end{array}$ & $\begin{array}{l}1-17 \\
\text { Jahre }\end{array}$ & & & allg. & $\begin{array}{l}\text { mit dieser } \\
\text { Hauptdiagnose }\end{array}$ \\
\hline 1 & M54 & Rückenschmerzen & 26,8 & 23,9 & 29,6 & 2,8 & 28,8 & 37,0 & 21,1 & 0,9 \\
\hline 2 & 110 & Essentielle (primäre) Hypertonie & 26,6 & 25,1 & 28,1 & 0,2 & 15,1 & 64,8 & 25,8 & 1,0 \\
\hline 3 & 106 & $\begin{array}{l}\text { Akute Infektionen an mehreren oder } \\
\text { nicht näher bezeichneten Lokalisationen } \\
\text { der oberen Atemwege }\end{array}$ & 22,0 & 21,6 & 22,3 & 39,8 & 23,4 & 9,0 & 13,2 & 0,3 \\
\hline 4 & $\mathrm{Z} 12$ & $\begin{array}{l}\text { Spezielle Verfahren zur Untersuchung } \\
\text { auf Neubildungen }\end{array}$ & 19,1 & 8,6 & 29,3 & 0,4 & 21,9 & 24,2 & 18,7 & 0,0 \\
\hline 5 & Zoo & $\begin{array}{l}\text { Allgemeinuntersuchung und Abklärung } \\
\text { bei Personen ohne Beschwerden oder } \\
\text { angegebene Diagnose }\end{array}$ & 18,0 & 17,0 & 18,9 & 32,4 & 11,2 & 21,1 & 16,8 & 0,0 \\
\hline 6 & E78 & $\begin{array}{l}\text { Störungen des Lipoproteinstoffwechsels } \\
\text { und sonstige Lipidämien }\end{array}$ & 16,1 & 15,7 & 16,5 & 0,1 & 8,9 & 39,6 & 25,2 & 0,0 \\
\hline 7 & Z25 & $\begin{array}{l}\text { Notwendigkeit der Impfung [Immuni- } \\
\text { sierung] gegen andere einzelne Virus- } \\
\text { krankheiten }\end{array}$ & 14,2 & 12,5 & 15,8 & 10,5 & 6,2 & 31,0 & 23,6 & 0,0 \\
\hline 8 & R10 & Bauch- und Beckenschmerzen & 11,2 & 7,2 & 15,0 & 10,2 & 12,8 & 8,6 & 25,2 & 1,5 \\
\hline 9 & E11 & Diabetes mellitus, Typ 2 & 10,1 & 10,2 & 10,0 & 0,0 & 4,3 & 27,3 & 29,9 & 2,0 \\
\hline 10 & N89 & $\begin{array}{l}\text { Sonstige nichtentzündliche Krankheiten } \\
\text { der Vagina }\end{array}$ & 9,9 & 0,0 & 19,5 & 1,5 & 14,9 & 5,1 & 18,6 & 0,0 \\
\hline 11 & E66 & Adipositas & 9,5 & 7,9 & 11,1 & 2,7 & 7,8 & 17,0 & 25,1 & 0,3 \\
\hline 12 & Z01 & $\begin{array}{l}\text { Sonstige spezielle Untersuchungen und } \\
\text { Abklärungen bei Personen ohne } \\
\text { Beschwerden oder angegebene Diagnose }\end{array}$ & 9,1 & 2,7 & 15,3 & 3,0 & 10,8 & 9,3 & 19,3 & 0,1 \\
\hline 13 & R52 & Schmerz, anderenorts nicht klassifiziert & 9,1 & 7,1 & 10,9 & 0,9 & 6,7 & 18,5 & 31,5 & 0,1 \\
\hline 14 & $\mathrm{~F} 32$ & Depressive Episode & 8,8 & 6,0 & 11,5 & 0,4 & 8,0 & 15,0 & 27,9 & 1,3 \\
\hline 15 & M17 & Gonarthrose [Arthrose des Kniegelenkes] & 8,8 & 6,9 & 10,5 & 0,0 & 4,1 & 23,0 & 28,5 & 2,5 \\
\hline 16 & A09 & $\begin{array}{l}\text { Sonstige und nicht näher bezeichnete } \\
\text { Gastroenteritis und Kolitis infektiösen und } \\
\text { nicht näher bezeichneten Ursprungs }\end{array}$ & 8,6 & 8,9 & 8,3 & 12,8 & 10,1 & 3,3 & 17,3 & 2,4 \\
\hline 17 & K29 & Gastritis und Duodenitis & 8,1 & 7,2 & 9,0 & 1,4 & 8,2 & 12,0 & 26,6 & 2,5 \\
\hline 18 & M99 & $\begin{array}{l}\text { Biomechanische Funktionsstörungen, } \\
\text { anderenorts nicht klassifiziert }\end{array}$ & 7,9 & 6,6 & 9,1 & 2,2 & 8,9 & 9,1 & 20,2 & 0,0 \\
\hline 19 & M25 & $\begin{array}{l}\text { Sonstige Gelenkkrankheiten, anderenorts } \\
\text { nicht klassifiziert }\end{array}$ & 7,7 & 6,9 & 8,5 & 2,3 & 7,9 & 10,5 & 23,1 & 0,5 \\
\hline 20 & M53 & $\begin{array}{l}\text { Sonstige Krankheiten der Wirbelsäule } \\
\text { und des Rückens, anderenorts nicht } \\
\text { klassifiziert }\end{array}$ & 7,6 & 5,9 & 9,4 & 0,5 & 7,4 & 12,2 & 22,7 & 0,4 \\
\hline 21 & $K_{21}$ & Gastroösophageale Refluxkrankheit & 7,6 & 7,0 & 8,1 & 0,3 & 6,0 & 14,9 & 27,3 & 0,8 \\
\hline 22 & M51 & Sonstige Bandscheibenschäden & 7,5 & 7,2 & 7,9 & 0,0 & 7,1 & 12,7 & 25,9 & 2,0 \\
\hline 23 & 120 & Akute Bronchitis & 7,3 & 7,2 & 7,4 & 14,1 & 6,2 & 5,7 & 18,5 & 2,0 \\
\hline 24 & E04 & Sonstige nichttoxische Struma & 7,3 & 3,6 & 10,9 & 0,3 & 5,8 & 14,3 & 22,4 & 0,7 \\
\hline 25 & $Z 27$ & $\begin{array}{l}\text { Notwendigkeit der Impfung [Immunisie- } \\
\text { rung] gegen Kombinationen von Infek- } \\
\text { tionskrankheiten }\end{array}$ & 7,2 & 6,9 & 7,5 & 17,4 & 4,6 & 5,1 & 15,3 & 0,0 \\
\hline 26 & L30 & Sonstige Dermatitis & 7,1 & 6,4 & 7,8 & 7,4 & 5,7 & 9,6 & 21,1 & 0,2 \\
\hline 27 & H52 & $\begin{array}{l}\text { Akkommodationsstörungen und } \\
\text { Refraktionsfehler }\end{array}$ & 6,9 & 5,9 & 8,0 & 6,5 & 2,8 & 15,6 & 23,3 & 0,0 \\
\hline 28 & 183 & Varizen der unteren Extremitäten & 6,9 & 4,0 & 9,8 & 0,0 & 4,5 & 15,7 & 25,7 & 1,2 \\
\hline 29 & Z30 & Kontrazeptive Maßnahmen & 6,9 & 0,0 & 13,5 & 2,2 & 11,7 & 0,1 & 15,6 & 0,0 \\
\hline 30 & M79 & $\begin{array}{l}\text { Sonstige Krankheiten des Weichteil- } \\
\text { gewebes, anderenorts nicht klassifiziert }\end{array}$ & 6,9 & 5,4 & 8,3 & 2,4 & 7,0 & 9,2 & 23,9 & 0,7 \\
\hline
\end{tabular}


2018 in stationärer Behandlung. Lediglich bei 1\% aller Patienten war die Diagnose zugleich der primäre Behandlungsanlass (stationäre Hauptdiagnose) für den Krankenhausaufenthalt.

Die häufigsten Behandlungsanlässe bei Kindern und Jugendlichen, jüngeren und älteren Erwachsenen sowie bei Männern und Frauen stehen elektronisch zur Verfügung. Von essentieller (primärer) Hypertonie und Rückenschmerzen waren vor allem Erwachsene betroffen. Während bei den jüngeren Erwachsenen (bis 59 Jahre) die Rückenschmerzen mit einer Prävalenz von $28,8 \%$ dominierten, war bei älteren Erwachsenen die essentielle (primäre Hypertonie) mit einer Prävalenz von $64,8 \%$ die häufigste dokumentierte Behandlungsdiagnose. Bei den Kindern und Jugendlichen (1-17 Jahre) dominierten wie auch in den Vorjahren akute Infektionen der oberen und unteren Atemwege wie beispielsweise akute Bronchitis (ICD-10 J20), akute Tonsillitis (ICD-10 Jo3) oder akute Pharyngitis (ICD-10 Jo3). In dieser Altersgruppe ist zudem auch die Prävalenz von Symptomen und abnormen klinischen und Laborbefunden, die anderenorts nicht klassifiziert sind, hoch (z.B. ICD-10 R50 Fieber sonstiger und unbekannter Ursache $[11,5 \%]$ und Rio Bauch- und Beckenschmerzen $[10,2 \%])$.

Frauen und Männer wiesen geschlechtsspezifische Besonderheiten in den Behandlungshäufigkeiten auf. So ist beispielsweise die dokumentierte Behandlungsprävalenz der depressiven Episode (ICD-1o F32) bei Frauen mit 11,5\% fast doppelt so hoch wie bei Männern mit 6,0\%. Auch spezielle Verfahren zur Untersuchung auf Neubildungen (ICD-10 Z12) wurden bei Frauen $(28,9 \%)$ deutlich häufiger dokumentiert als bei Männern (8,6\%).

In Ergänzung hierzu stehen im elektronischen Anhang Prävalenzen und Hospitalisierungsraten des Jahres 2018 auf Ebene der ICD10-Diagnoseobergruppen zur Verfügung. Mit einer Prävalenz von 37,2\% stellte die Diagnosegruppe Zoo-Z13 (Personen, die das Gesundheitswesen zur Untersuchung und Abklärung in Anspruch nehmen) den häufigsten Behandlungsanlass der deutschen Wohnbevölkerung im Jahr
2018 dar. In diese Obergruppe fallen beispielsweise Früherkennungs- und Reihenuntersuchungen sowie Nachuntersuchungen nach Behandlung von bösartigen Neubildungen. Bei Frauen war die Prävalenz mehr als doppelt so hoch wie bei Männern, was durch eine höhere Teilnahme von Frauen an Früherkennungsuntersuchungen zu erklären sein dürfte (Tillmanns et al. 2019). Bei Betrachtung der Behandlungsprävalenzen auf der Ebene der Diagnosegruppen wird insbesondere auch die hohe Prävalenz der dokumentierten Celenkerkrankungen (Arthropathien) deutlich. Mehr als ein Viertel der Bevölkerung (26,2\%) hatte eine Diagnose aus dem Bereich der Arthropathien (Moo-M25). In der Altersgruppe der über 6o-Jährigen war fast die Hälfte der Bevölkerung (49\%) betroffen.

\subsection{Inanspruchnahme innerhalb der einzelnen Leistungssektoren}

\subsubsection{Stationäre Behandlungen}

Im Jahr 2018 wurden von 100.00o Einwohnern insgesamt 15.353 Personen mindestens einmal stationär behandelt (s. Abb. 3). Innerhalb der drei Altersgruppen sind erwartungsgemäß erhebliche Unterschiede in der Inanspruchnahme von stationären Leistungen zu sehen. In der Altersgruppe der über 6o-Jährigen hatte jede vierte Person mindestens eine stationäre Behandlung. Ältere Personen wurden damit mehr als doppelt so oft in einer Klinik behandelt wie Erwachsene der Altersgruppe 18 bis 59 Jahre und etwa dreimal häufiger als Kinder und Jugendliche.

Bei der Inanspruchnahme von stationären Leistungen gab es große regionale Unterschiede (s. Abb. 4). Die Regionen Emscher-Lippe (19.604 Patienten je 100.000 Einwohner) und Arnsberg (18.699 Patienten je 100.000 Einwohner) in Nordrhein-Westfalen sowie die Region Altmark (18.228 Patienten je 100.000 Einwohner) in Sachsen-Anhalt wiesen den höchsten Anteil an Krankenhauspatienten auf. Besonders niedrig war die Hospitalisierungsrate in weiten Teilen 


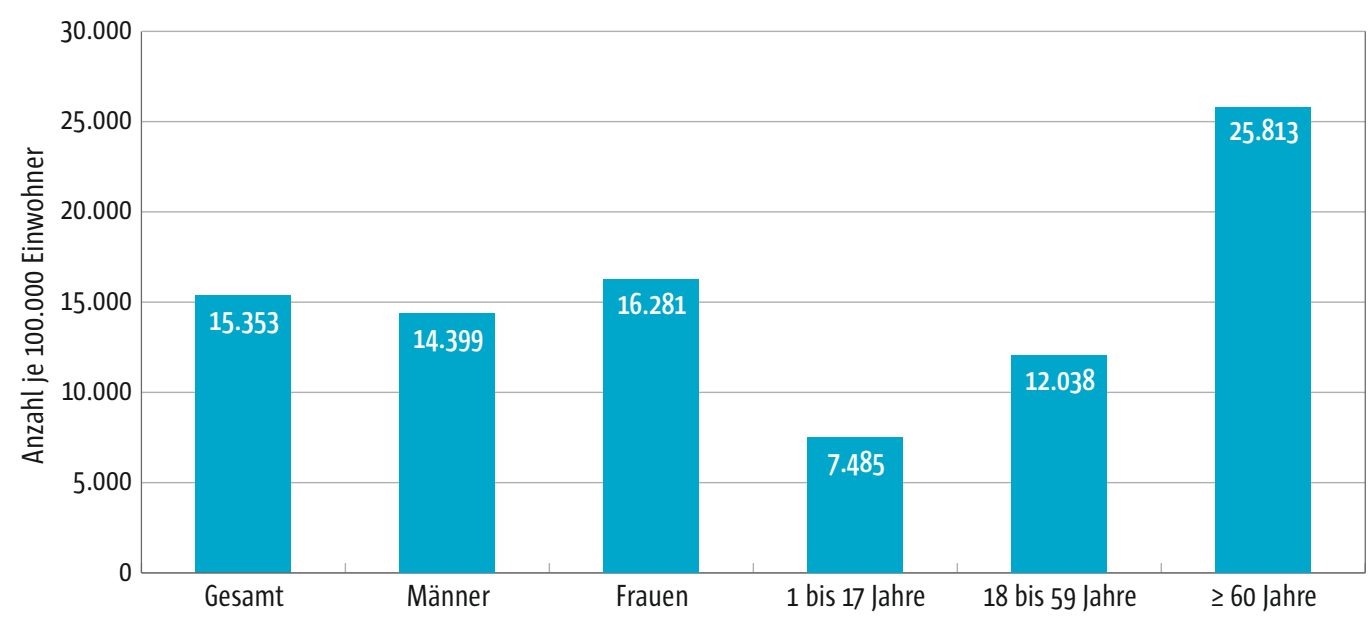

Abb. 3 Anzahl der jährlichen Krankenhauspatienten je 100.000 Einwohner nach Alter und Geschlecht (2018)

Baden-Württembergs (Minimum in der Region Neckar-Alb mit 12.603 Krankenhauspatienten je 100.000 Einwohner).

Das Statistische Bundesamt veröffentlicht jährlich mit der DRG-Statistik Zahlen zur Entwicklung der (vollstationären) Fälle in deutschen Krankenhäusern. Der Statistik nach sind die absoluten Fallzahlen im Zeitraum 2007 bis 2016 erheblich (insgesamt um 12,5\%) gestiegen (Statistisches Bundesamt 2016). Zwischen 2016 und 2018 ist ein leichter Rückgang $(-1,1 \%)$ der Fallzahlen zu beobachten (Statistisches Bundesamt 2021b).

\section{Die häufigsten Behandlungsanlässe}

Die 30 häufigsten stationären Behandlungsanlässe aller im Jahr 2018 abgeschlossenen vollund teilstationären Krankenhausfälle sind in Tabelle 2 dargestellt. Eine erweiterte Tabelle über die 100 häufigsten stationären Behandlungsdiagnosen ist elektronisch verfügbar. Für jede Patientin/jeden Patienten wird in dieser Tabelle nur die Diagnose ausgewertet, die hauptsächlich für die Veranlassung des stationären Krankenhausaufenthaltes verantwortlich ist (Hauptdiagnose).

\section{Tabelle 2: Die Bedeutung der Tabellenspalten im Einzelnen}

- Rang: Rangposition in der „Hitliste“ der häufigsten stationären Behandlungsanlässe des Jahres 2018

- ICD-10: Dreistellige ICD-Schlüsselnummer nach ICD10-GM. Es wurden auch ICD-Schlüsselnummern eingeschlossen, die zur Inanspruchnahme des Gesundheitswesens führen und nicht als Krankheit oder Verletzung klassifizierbar sind („Z-Diagnosen“).

- Diagnose bzw. Behandlungsanlass: Klartextbeschreibung der ICD-Schlüsselnummer

- KH-Patienten je 100.000 Einwohner: Anzahl der Personen in der Gesamtbevölkerung, die - von 100.000 Einwohnern - im Jahr mindestens einen Krankenhausaufenthalt anlässlich der betreffenden Diagnose hatten.

v Fälle je KH-Patient: durchschnittliche Anzahl an Krankenhausfällen je stationär behandeltem Patient

Zu den häufigsten stationären Behandlungsanlässen des Jahres 2018 zählten unverändert Geburten (ICD-10 Z38), Herzinsuffizienz (ICD-10 I50), psychische und Verhaltensstörungen durch Alkohol (ICD-10 F10), Angina pectoris (ICD-10 I20) und Pneumonie (ICD-10 J18). Diese fünf Behandlungsanlässe führten bereits in den früheren Jahren die Liste der wichtigsten Hauptdiagnosen stationärer Aufenthalten an. 


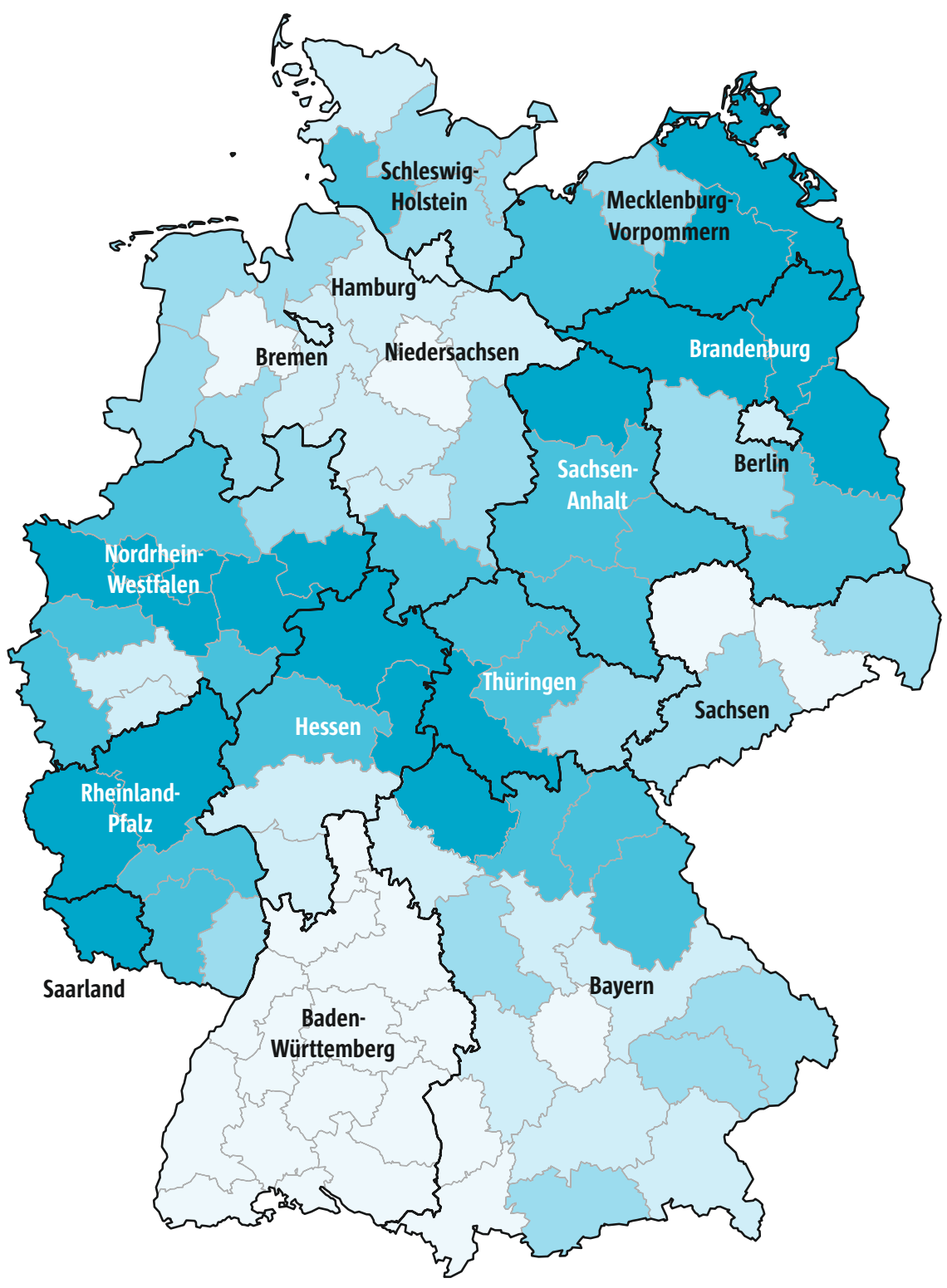

Anzahl der jährlichen Krankenhauspatienten je 100.000 Einwohner*
$\square$ 12.603-14.683
$\square$ 14.684-15.548
$\square$ 15.549-16.094
$16.095-17.257$
$17.258-19.604$

* standardisiert auf die deutsche Wohnbevölkerung

Abb. 4 Anzahl der jährlichen Krankenhauspatienten je 100.000 Einwohner nach Raumordnungsregionen (2018) 
17 Diagnosehäufigkeit und Inanspruchnahme von Gesundheitsleistungen

Tab. 2 Die 30 häufigsten stationären Behandlungsanlässe des Jahres 2018 bei Krankenhauspatienten in Deutschland

\begin{tabular}{|c|c|c|c|c|}
\hline \multirow[b]{2}{*}{ Rang } & \multicolumn{2}{|c|}{ Hauptdiagnose } & \multicolumn{2}{|c|}{ KH-Patienten } \\
\hline & $\begin{array}{l}\text { ICD-10 } \\
\text { (Dreisteller) }\end{array}$ & Diagnose bzw. Behandlungsanlass & $\begin{array}{l}\text { je } 100.000 \\
\text { Einwohner }\end{array}$ & $\begin{array}{l}\text { Fälle je } \\
\text { KH-Patient }\end{array}$ \\
\hline & insgesamt & & 15.353 & 1,6 \\
\hline 1 & Z38 & Lebendgeborene nach dem Geburtsort & 678 & 1,0 \\
\hline 2 & 150 & Herzinsuffizienz & 503 & 1,3 \\
\hline 3 & |18 & Pneumonie, Erreger nicht näher bezeichnet & 294 & 1,1 \\
\hline 4 & F10 & Psychische und Verhaltensstörungen durch Alkohol & 287 & 1,7 \\
\hline 5 & So6 & Intrakranielle Verletzung & 278 & 1,1 \\
\hline 6 & 148 & Vorhofflimmern und Vorhofflattern & 275 & 1,2 \\
\hline 7 & 163 & Hirninfarkt & 274 & 1,2 \\
\hline 8 & 120 & Angina pectoris & 271 & 1,1 \\
\hline 9 & 144 & Sonstige chronische obstruktive Lungenkrankheit & 268 & 1,5 \\
\hline 10 & M54 & Rückenschmerzen & 257 & 1,1 \\
\hline 11 & K80 & Cholelithiasis & 256 & 1,2 \\
\hline 12 & $\mid 10$ & Essentielle (primäre) Hypertonie & 255 & 1,1 \\
\hline 13 & 121 & Akuter Myokardinfarkt & 240 & 1,2 \\
\hline 14 & M17 & Gonarthrose [Arthrose des Kniegelenkes] & 221 & 1,0 \\
\hline 15 & 170 & Atherosklerose & 219 & 1,3 \\
\hline 16 & E11 & Diabetes mellitus, Typ 2 & 209 & 1,2 \\
\hline 17 & A09 & $\begin{array}{l}\text { Sonstige und nicht näher bezeichnete Gastroenteritis und Kolitis } \\
\text { infektiösen und nicht näher bezeichneten Ursprungs }\end{array}$ & 207 & 1,0 \\
\hline 18 & 125 & Chronische ischämische Herzkrankheit & 206 & 1,1 \\
\hline 19 & K29 & Gastritis und Duodenitis & 201 & 1,0 \\
\hline 20 & F33 & Rezidivierende depressive Störung & 193 & 1,2 \\
\hline 21 & S72 & Fraktur des Femurs & 189 & 1,1 \\
\hline 22 & N39 & Sonstige Krankheiten des Harnsystems & 189 & 1,1 \\
\hline 23 & K40 & Hernia inguinalis & 186 & 1,0 \\
\hline 24 & R07 & Hals- und Brustschmerzen & 183 & 1,0 \\
\hline 25 & R55 & Synkope und Kollaps & 179 & 1,0 \\
\hline 26 & M16 & Koxarthrose [Arthrose des Hüftgelenkes] & 178 & 1,0 \\
\hline 27 & 080 & Spontangeburt eines Einlings & 172 & 1,0 \\
\hline 28 & R10 & Bauch- und Beckenschmerzen & 170 & 1,0 \\
\hline 29 & E86 & Volumenmangel & 169 & 1,1 \\
\hline 30 & A41 & Sonstige Sepsis & 163 & 1,1 \\
\hline
\end{tabular}




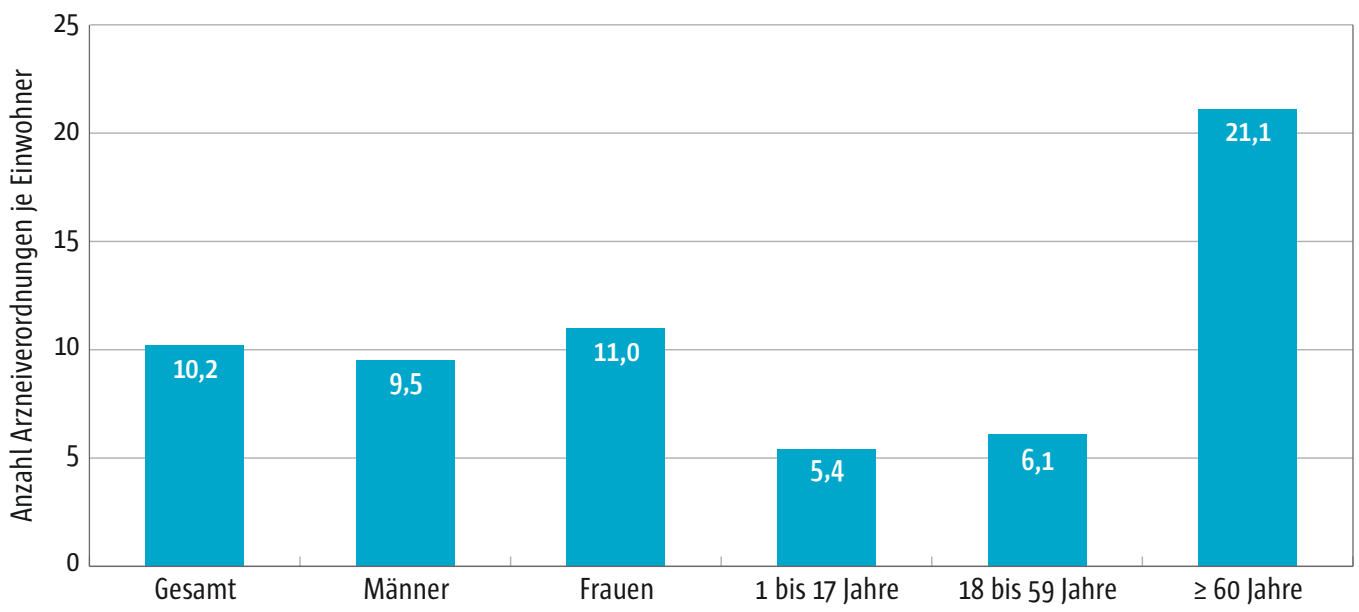

Abb. 5 Anzahl der jährlichen Arzneiverordnungen je Einwohner nach Geschlecht und Alter (2018)

Allerdings ist die Häufigkeit insgesamt im Niveau gestiegen. Beispielsweise ist die Zahl der Krankenhauspatienten je 100.00o Einwohner mit Hauptdiagnose Herzinsuffizienz (ICD10 I5o) von 347 im Jahr 2010 auf 503 im Jahr 2018 angestiegen (Gerste et al. 2012; Schmuker et al . 2019).

Auf alle Personen der deutschen Wohnbevölkerung, die 2018 stationär behandelt wurden, entfielen im Mittel 1,6 Krankenhausfälle. Das bedeutet, dass ein Croßteil der Patienten im Jahr 2018 mehr als einmal in ein Krankenhaus aufgenommen wurde. Die Anzahl der Krankenhausfälle je Patient schwankte jedoch in Abhängigkeit von der Hauptdiagnose. Bösartige Neubildungen der Bronchien und der Lungen (ICD-10 C34), der Harnblase (ICD-10 C67) und des Kolons (ICD-10 C18) waren am häufigsten für mehrfache stationäre Aufenthalte verantwortlich, gefolgt von psychischen und Verhaltensstörungen durch Alkohol (ICD-10 F1o).

\subsubsection{Arzneiverordnungen}

Im Jahr 2018 haben mit 77,1\% über drei Viertel der deutschen Bevölkerung mindestens eine Verordnung für ein erstattungsfähiges Arznei- mittel erhalten. Auf jeden dieser Arzneimittelpatienten entfielen im Mittel 13,4 Verordnungen und rein rechnerisch im Durchschnitt 804 Tagesdosen mindestens eines Arzneimittels (vgl. Wirkstoffgruppen gemäß ATC-Klassifikation im elektronischer Anhang).

Bezogen auf die Gesamtbevölkerung wurden im Jahr 2018 je Einwohner im Mittel 10,2 Arzneimittel verordnet (s. Abb. 5). Die Häufigkeit von Arzneiverordnungen variiert in Abhängigkeit vom Alter erkennbar. Während es bei Kinder und Jugendlichen ( 1 bis 17 Jahren) und bei Erwachsenen mittleren Alters (18 bis 59 Jahren) ca. 6 jährliche Verordnungen waren, erhielten ältere Personen durchschnittlich 21,1 Arzneiverordnungen.

In Abhängigkeit von der geografischen Region streute die Häufigkeit von Arzneiverordnungen im Jahr 2018 deutlich (s. Abb. 6). Mit mehr als 13,6 Verordnungen pro Einwohner lagen die Regionen Vorpommern, Westmecklenburg und Saar an der Spitze des Verordnungsgeschehens. Die niedrigsten Raten $(<8,5$ Verordnungen je Einwohner) wurden in den Bundesländern Bayern und Baden-Württemberg dokumentiert und hier speziell in den Regionen Allgäu, Oberland, München, Südostoberbayern und Donau-Iller. 


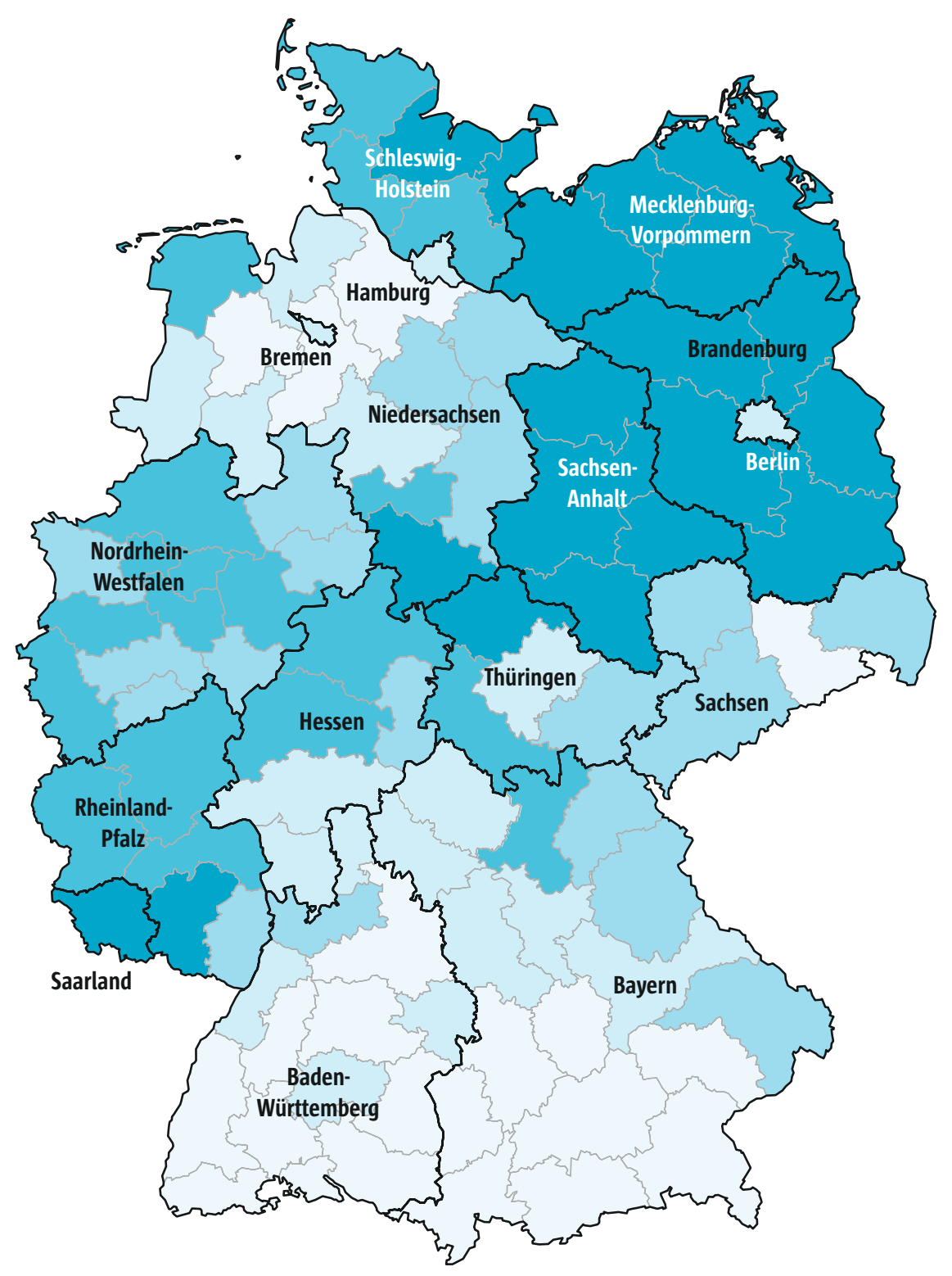

Anzahl der jährlichen Arzneiverordnungen je Einwohner *
$\square 7,22-8,46$
$\square$ 8,47-9,18
$\square$ 9,19-9,67
$9,68-10,44$
$10,44-13,64$

* standardisiert auf die deutsche Wohnbevölkerung

Abb. 6 Anzahl der jährlichen Arzneiverordnungen je Einwohner nach Raumordnungsregionen (2018) 


\section{Arzneiverordnungen nach therapeutischer Wirkstoffgruppe}

\section{Abbildung 7: Die Bedeutung der Kennziffern im Einzelnen}

Wirkstoffgruppe mit ATC-Code: Zweite hierarchische Ebene des anatomisch-therapeutisch-chemischen Klassifikationssystems (ATC): therapeutische Untergruppe mit dreistelligem ATC-Code. Eine verlässliche Zuordnung der Arzneimittel zu ATC-Gruppen samt Bestimmung der verordneten Tagesdosen in DDD kann nur für Fertigarzneimittel vorgenommen werden, die entsprechend klassifiziert sind.

- Verordnungsrate: Verordnungsrate je ATC-Gruppe. Anteil der Personen in der Gesamtbevölkerung, die im Jahr mindestens eine Verordnung aus der betreffenden Wirkstoffgruppe erhalten haben.

- Verordnungen je Arzneimittelpatient der ATC-Gruppe: durchschnittliche Anzahl an Verordnungen, die ein Patient mit Verordnung in der betreffenden Wirkstoffgruppe erhalten hat

- Tagesdosen (DDD) je Arzneimittelpatient der ATCGruppe: verordnete Arzneimittelmenge (Angabe in DDD) je Patient

Einen Überblick über die häufigsten im Jahr 2018 verordneten Wirkstoffgruppen gemäß ATC-Klassifikation liefert Abbildung 7. Dargestellt wurde der prozentuale Anteil an Personen mit einer Verordnung der jeweiligen Wirkstoffgruppe bezogen auf die deutsche Wohnbevölkerung. Die ebenso dargestellten definierten Tagesdosen (DDD) beziehen sich dagegen ausschließlich auf diejenigen Patienten, die tatsächlich eine entsprechende Medikation verordnet bekamen. Die DDD wird dabei als Maß für die verordnete Arzneimittelmenge verwendet. Wie auch in früheren Jahren wurden sowie Antiphlogistika und Antirheumatika (ATC Mo1) sowie Antibiotika zur systemischen Anwendung (ATC Jo1)besonders häufig verordnet (Gerste et al. 2016; Schmuker et al. 2019). Fast ein Drittel der Deutschen hat 2018 mindestens eine solche Verordnung erhalten. Je Arzneimittelpatient wurden im Mittel 1,7 Packungen
Antibiotika mit 15 Tagesdosen verordnet, bei den Antiphlogistika und Antirheumatika waren es 2,o Packungen mit 55 DDD. Die vergleichsweise niedrigen DDD-Mengen bei den Antibiotika weisen darauf hin, dass diese beiden Wirkstoffgruppen primär bzw. häufig zur Behandlung akuter oder schubweiser Krankheitsverläufe eingesetzt werden. Anders verhält es sich bei chronischen Erkrankungen wie Hypertonie oder Diabetes mellitus, die einen kontinuierlichen Behandlungsbedarf erfordern. Dementsprechend hoch sind die Tagesdosen bei Mitteln mit Wirkung auf das ReninAngiotensin-System (ATC Co9; 581 DDD je Arzneimittelpatient) oder bei den Antidiabetika (ATC A1o; 468 DDD je Arzneimittelpatient). Insgesamt ist im GKV-Arzneimittelmarkt ein kontinuierlicher Anstieg des Verordnungsvolumens nach definierten Tagesdosen (DDD) zu beobachten. Zwischen 2008 und 2018 sind die definierten Tagesdosen im Gesamtmarkt (Generika und patentgeschützte Arzneimittel) von 32,3 Mrd. auf 41,4 Mrd. und damit um fast 30\% gestiegen (Schwabe et al. 2018).

\subsection{3 Ärztliche Inanspruchnahme}

Im Jahr 2018 haben 9o, 5\% der deutschen Wohnbevölkerung mindestens einmal einen ambulant tätigen Vertragsarzt aufgesucht (vgl. elektronischer Anhang). Bezogen auf die Gesamtbevölkerung entfielen auf jede Person durchschnittlich 8,1 ambulante Behandlungsfälle (s. Abb. 8). Dabei gelten mehrere Praxisbesuche eines Patienten pro Quartal bei ein und demselben Arzt als ein einziger Behandlungsfall. Im Mittel wurden pro Person und Quartal demzufolge mehr als zwei ambulant tätige Vertragsärzte aufgesucht. Erwartungsgemäß war die Inanspruchnahme ambulanter Leistungen bei älteren Erwachsenen der Altersgruppe (6o Jahre und älter) am höchsten (11,6 Behandlungsfälle je Einwohner).

Die kartografische Darstellung ambulanter Behandlungsfälle je Einwohner zeigte eine 


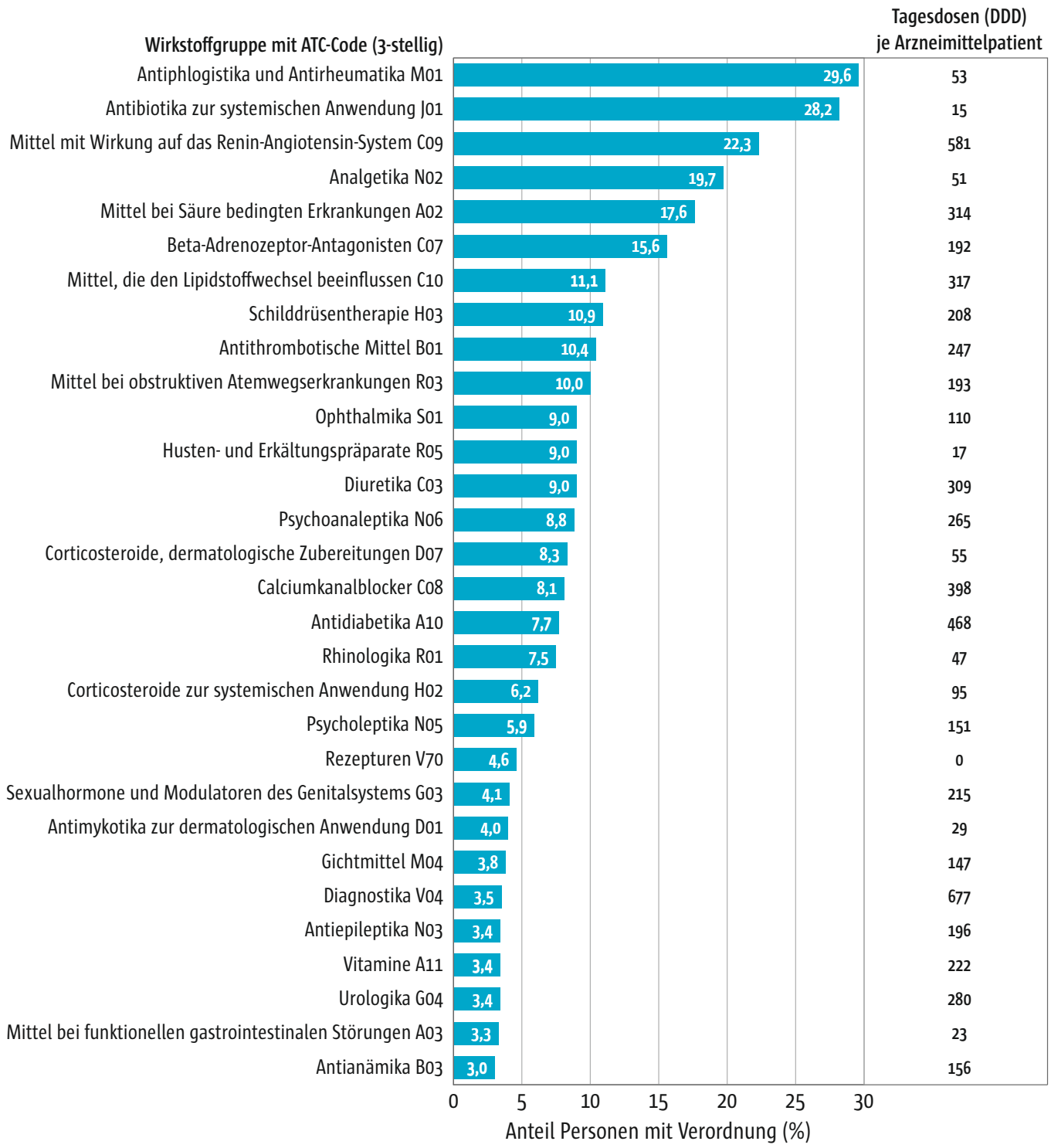

Abb. 7 Die am häufigsten verordneten Wirkstoffgruppen in Deutschland (2018)

leicht überdurchschnittliche Konsultation ambulanter Ärzte in Nordost- und Mitteldeutschland (s. Abb. 9). So lagen die Regionen Oberfranken und Würzburg sowie mittleres Mecklenburg/Rostock mit mehr als 8,8 Behandlungsfällen je Einwohner an der Spitze der ambulanten ärztlichen Inanspruchnahme. Weniger als 7,4 Behandlungsfälle je Einwohner wurden in den Regionen Arnsberg (Nordrhein-Westfalen) und Altmark (Sachsen-Anhalt) dokumentiert. Diese Regionen wiesen also die geringste ambulante Behandlungshäufigkeit auf, während sie zugleich als Regionen mit besonders hoher Anzahl stationärer Aufenthalte je 100.00o Einwohner auffielen. 


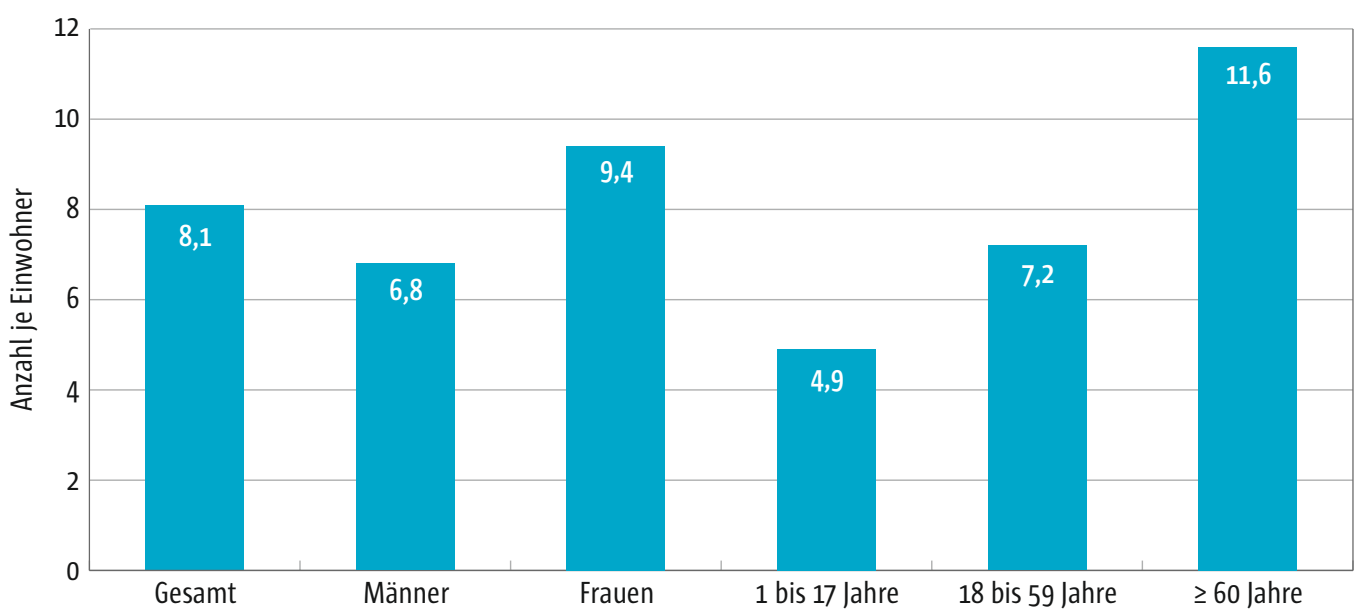

Abb. 8 Anzahl der jährlichen ambulanten Behandlungsfälle je Einwohner nach Alter und Geschlecht (2018)

Die ärztliche Inanspruchnahme differenziert nach EBM-Facharztgruppen bzw. ärztlichen Versorgungsbereichen zeigt Tabelle 3 (zur Methodik s. Kap. 17.2.1). Mehr als Dreiviertel der Bevölkerung $(76,4 \%)$ nahm eine hausärztliche Versorgung in Anspruch. Bei älteren Erwachsenen (ab Go Jahren) lag die hausärztliche Behandlungsrate noch deutlich höher $(92,6 \%)$. Kinder und Jugendliche waren mit einer Behandlungsrate von 69,2\% erwartungsgemäß vorrangig beim Kinderarzt. Etwa ein Fünftel der Bevölkerung (19,1\%) wurde notfallärztlich über den kassenärztlichen Bereitschaftsdienst oder die Notfallambulanzen der Krankenhäuser versorgt. Kinder und Jugendliche nahmen die Notfallversorgung überdurchschnittlich häufig (28,4 \%) in Anspruch. In Ergänzung hierzu zeigt Abbildung 10 die Verteilung aller ambulanten Behandlungsfälle in Deutschland auf die EBM-Arztfachgruppen sowie die durchschnittliche Anzahl an behandelten Fällen je Gruppe. Etwa ein Drittel der gesamten Behandlungsfälle entfallen demnach auf die hausärztliche Versorgung (32,2\%). An der hausärztlichen Versorgung beteiligte Ärzte haben im Durchschnitt je Einwohner 2,7 Behandlungsfälle behandelt, in den anderen Arztfachgruppen bzw. Versorgungsbereichen liegt der Anteil der Behandlungsfälle je Einwohner deutlich darunter.

\section{Tabelle 3: Die Bedeutung der Tabellenspalten} im Einzelnen

- Pauschale: Facharztgruppen des Einheitlichen Bewertungsmaßstabs (EBM) mit Versicherten- und Grundpauschalen sowie die ambulante Notfallversorgung (gem. Kapitel 1.2 des EBM) sowie den ihnen gleichgestellten und regional vereinbarten ambulanten Notfallleistungen

- Behandlungsrate: Anteil der Personen in der Bevölkerung, bei denen im Jahr mindestens einmal die jeweilige Pauschale abgerechnet wurde

- Behandlungsrate männlich: Anteil der männlichen Personen, die im Jahr mindestens einmal die jeweilige Fachgruppe (bzw. Versorgung) in Anspruch genommen haben

- Behandlungsrate weiblich: Anteil der weiblichen Personen, die im Jahr mindestens einmal die jeweilige Fachgruppe (bzw. Versorgung) in Anspruch genommen haben

- Behandlungsrate 1 bis 17 Jahre: Anteil aller Kinder und Jugendlicher in der Bevölkerung, die im Jahr mindestens einmal die jeweilige Fachgruppe (bzw. Versorgung) in Anspruch genommen haben

- Behandlungsrate 18 bis 59 Jahre: Anteil aller Personen im Alter von 18 bis 59 Jahren, die im Jahr mindestens einmal die jeweilige Fachgruppe (bzw. Versorgung) in Anspruch genommen haben 


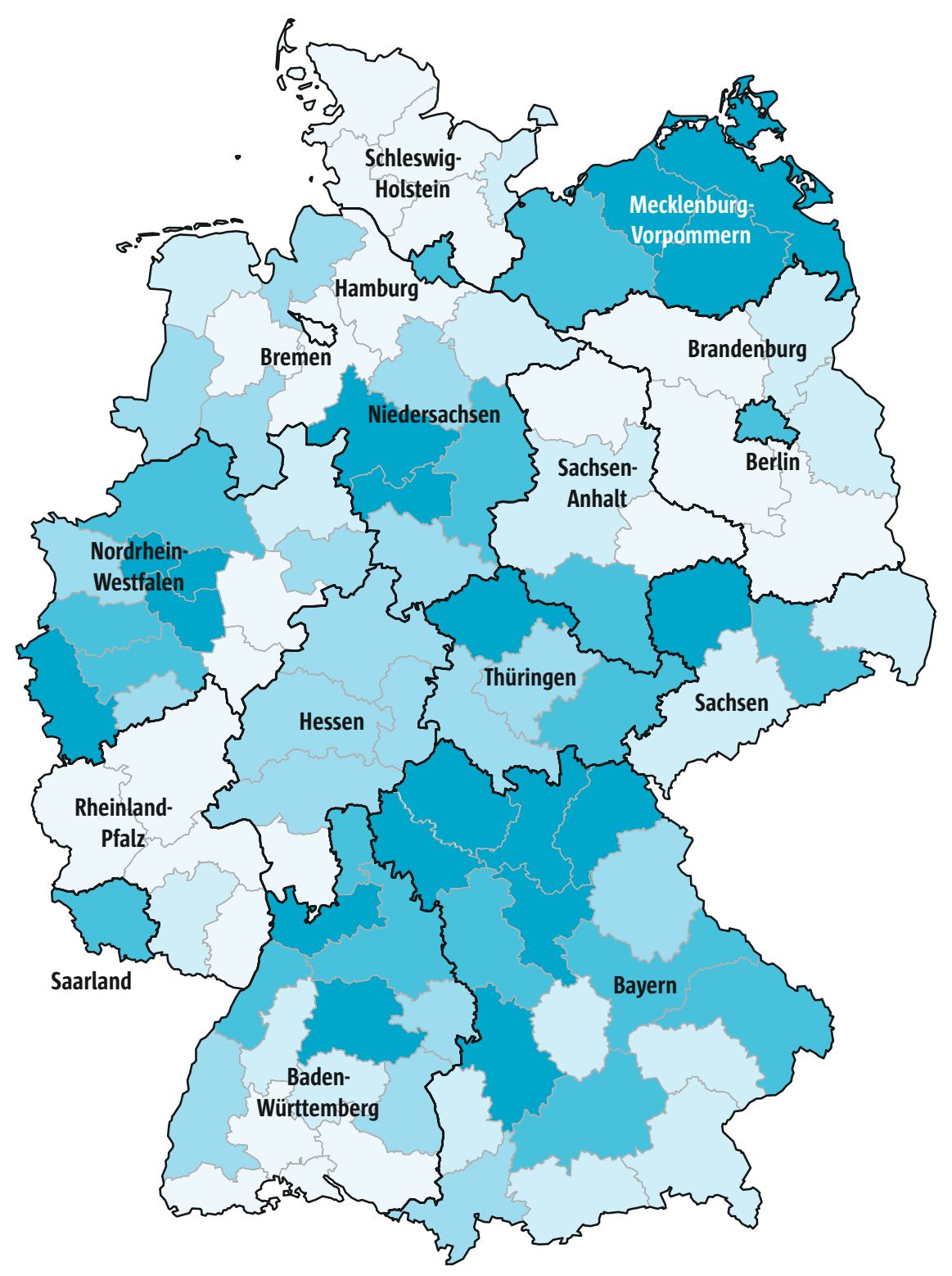

Anzahl der jährlichen ambulanten Behandlungsfälle je Einwohner*
$\square 7,3-7,86$
$\square 7,87-8,01$
$\square$ 8,02-8,20
$8,21-8,38$
$8,39-8,80$

* standardisiert auf die deutsche Wohnbevölkerung

Abb. 9 Anzahl der jährlichen ambulanten Behandlungsfälle je Einwohner nach Raumordnungsregionen (2018) 
Tab. 3 Ärztliche Inanspruchnahme nach Grund- und Versichertenpauschale (2018)

\begin{tabular}{|c|c|c|c|c|c|c|}
\hline Pauschale* & gesamt & männlich & weiblich & $\begin{array}{l}1-17 \\
\text { Jahre }\end{array}$ & $\begin{array}{l}\text { 18-59 } \\
\text { Jahre }\end{array}$ & $\begin{array}{l}60 \text { und } \\
\text { mehr Jahre }\end{array}$ \\
\hline Hausärzte & 76,4 & 73,1 & 79,6 & 36,3 & 80,3 & 92,6 \\
\hline Internisten & 17,1 & 16,2 & 17,9 & 1,3 & 13,6 & 32,9 \\
\hline Kinderärzte & 11,8 & 12,2 & 11,3 & 69,2 & 0,5 & 0,0 \\
\hline Gynäkologen & 20,8 & 0,3 & 40,8 & 4,3 & 28,4 & 15,6 \\
\hline HNO-Ärzte & 15,8 & 14,7 & 16,9 & 14,6 & 13,5 & 21,1 \\
\hline Augenärzte & 21,5 & 18,6 & 24,4 & 21,8 & 13,2 & 38,2 \\
\hline Chirurgen & 11,4 & 11,0 & 11,7 & 6,4 & 11,6 & 13,8 \\
\hline Orthopäden & 18,4 & 15,6 & 21,2 & 9,3 & 18,0 & 24,2 \\
\hline Urologen & 8,1 & 12,2 & 4,1 & 1,4 & 5,6 & 16,9 \\
\hline Hautärzte & 14,2 & 12,0 & 16,3 & 9,7 & 13,7 & 17,8 \\
\hline Nervenärzte & 10,3 & 8,8 & 11,7 & 3,4 & 9,6 & 15,6 \\
\hline sonstige & 13,5 & 9,1 & 17,8 & 9,0 & 12,9 & 17,4 \\
\hline Radiologen, Strahlentherapeuten, Nuklearmediziner & 15,9 & 13,3 & 18,4 & 2,8 & 16,0 & 23,3 \\
\hline Notfall & 19,1 & 18,3 & 19,9 & 28,4 & 18,2 & 15,7 \\
\hline Labormediziner & 40,8 & 30,7 & 50,6 & 17,2 & 41,7 & 52,9 \\
\hline $\begin{array}{l}\text { weitere vertragsärztliche Leistungen ohne } \\
\text { Versicherten-/Grundpauschale }\end{array}$ & 49,3 & 37,1 & 61,1 & 19,6 & 49,9 & 65,3 \\
\hline gesamt & 90,5 & 87,1 & 93,8 & 92,9 & 87,4 & 95,1 \\
\hline
\end{tabular}

*Facharztgruppen des Einheitlichen Bewertungsmaßstabs (EBM) mit Versicherten- und Grundpauschalen sowie die ambulante Notfallversorgung (gem. Kapitel 1.2 des EBM) sowie den ihnen gleichgestellten und regional vereinbarten ambulanten Notfallleistungen

Behandlungsrate 60 und mehr Jahre: Anteil aller Personen im Alter 60 Jahre und älter in der Bevölkerung, die im Jahr mindestens einmal die jeweilige Fachgruppe (bzw. Versorgung) in Anspruch genommen haben

\subsubsection{Inanspruchnahme von Heilmitteln}

Im Jahr 2018 nahmen von 100.00o Einwohnern mehr als 18.309 Personen eine Heilmittelbehandlung in Anspruch (s. Abb. 11). Bei den Frauen lag der Anteil der Heilmittelverordnungen (21.643 Verordnungen je 100.000 weibliche Einwohner) deutlich höher als bei Männern
(14.880 Verordnungen je 100.000 männliche Einwohner). Die Inanspruchnahme war bei Kindern und Jugendlichen der Altersklasse 1 bis 17 Jahre am geringsten und stieg mit dem Alter. Das Maximum wurde bei Personen erreicht, die 6o Jahre oder älter waren (29.159 Verordnungen je 100.00o Einwohner).

Die regionalen Unterschiede in den Behandlungsraten je 100.00o Einwohner in Deutschland zeigt Abbildung 12. Die höchsten Behandlungsraten mit über 20.00o Heilmittelpatienten im Jahr 2018 weisen Regionen aus den östlichen Bundesländern auf (z.B. OberlausitzNiederschlesien 26.792/100.00o Einwohner). Dagegen lagen die Behandlungsraten in 


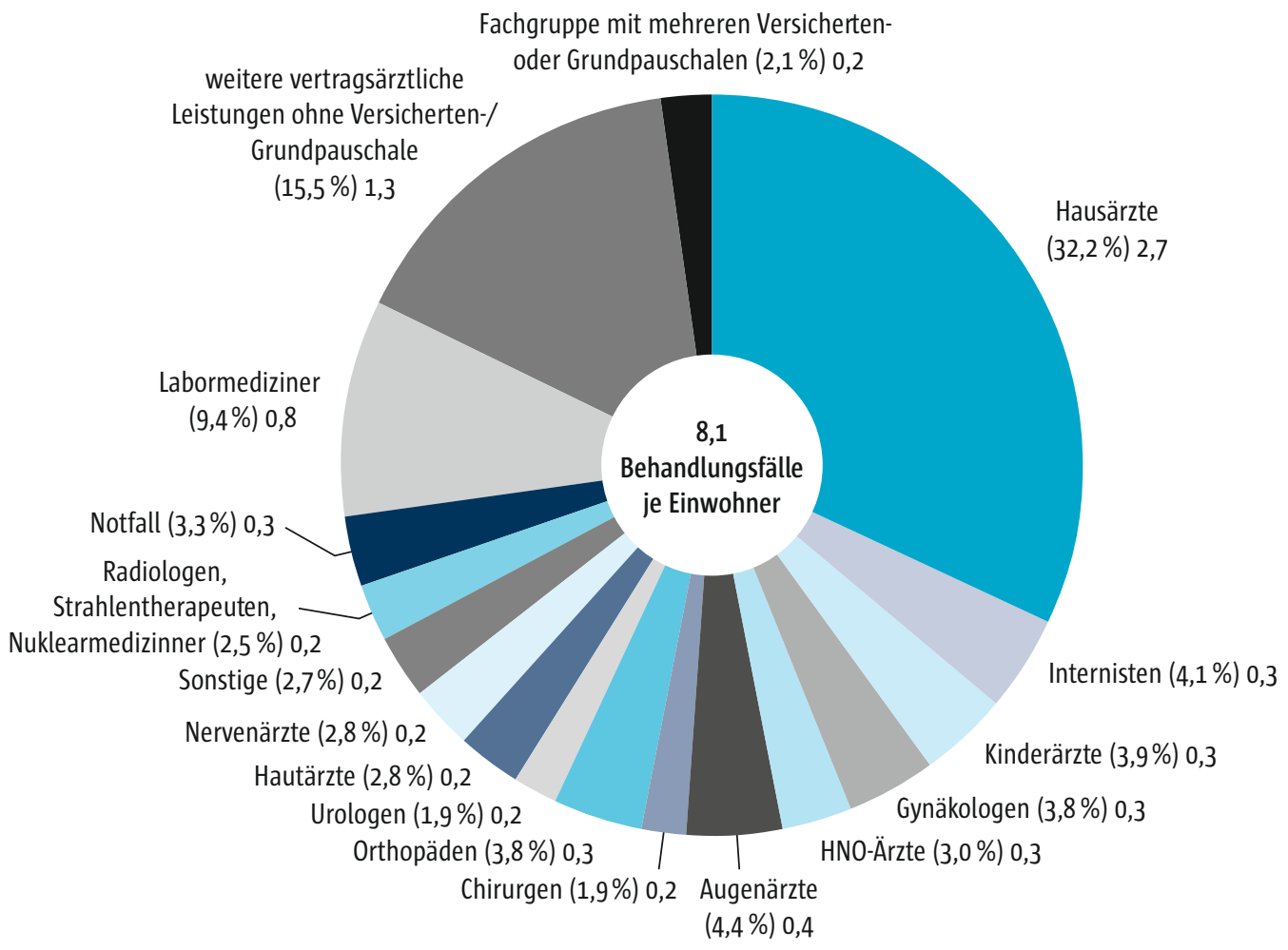

Behandlungsfälle je Einwohner: Die Anzahl von Behandlungsfällen bezogen auf alle Einwohner dargestellt, unabhängig davon, ob sie tatsächlich beim Arzt waren und selbst einen Behandlungsfall verursacht haben oder nicht.

Anteil Fälle je Einwohner: Die Anzahl der Behandlungsälle je Fachgruppe summiert sich zur Anzahl der Fälle gesamt und wird hier als Anteilswert an allen Behandlungsfällen ausgedrückt.

Abb. 10 Ambulante Behandlungsfälle je Einwohner nach EBM-Facharztgruppen (2018)

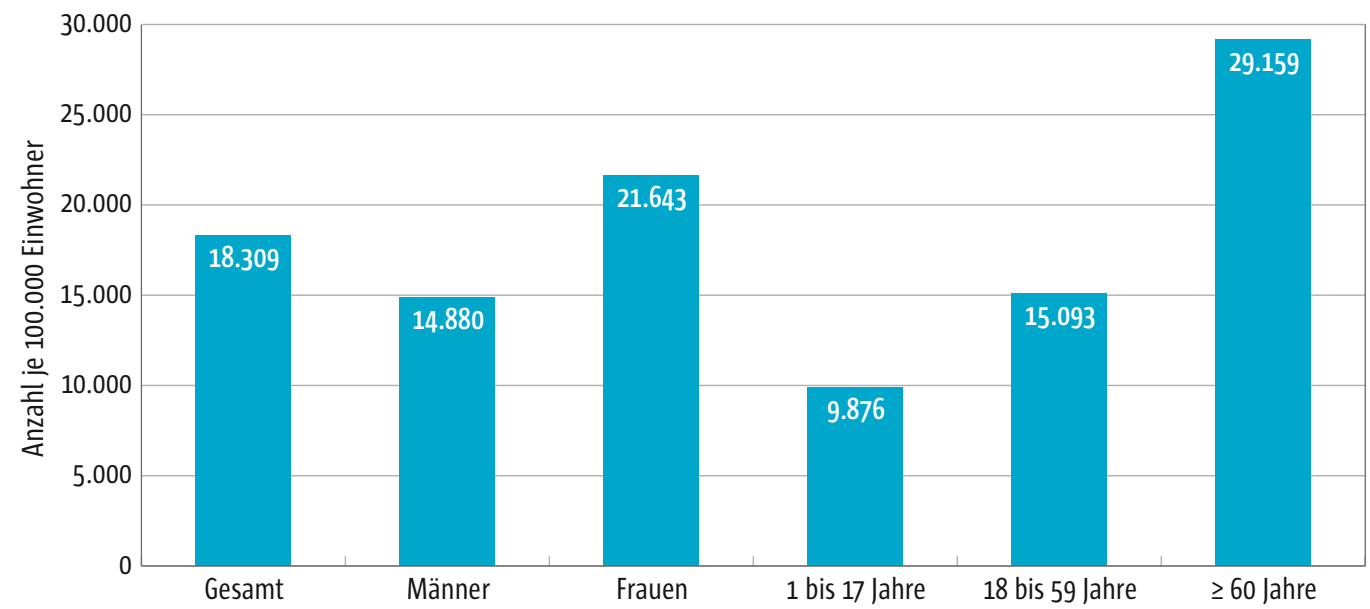

Abb. 11 Anzahl der jährlichen Heilmittelpatienten je 100.000 Einwohner nach Alter und Geschlecht (2018) 


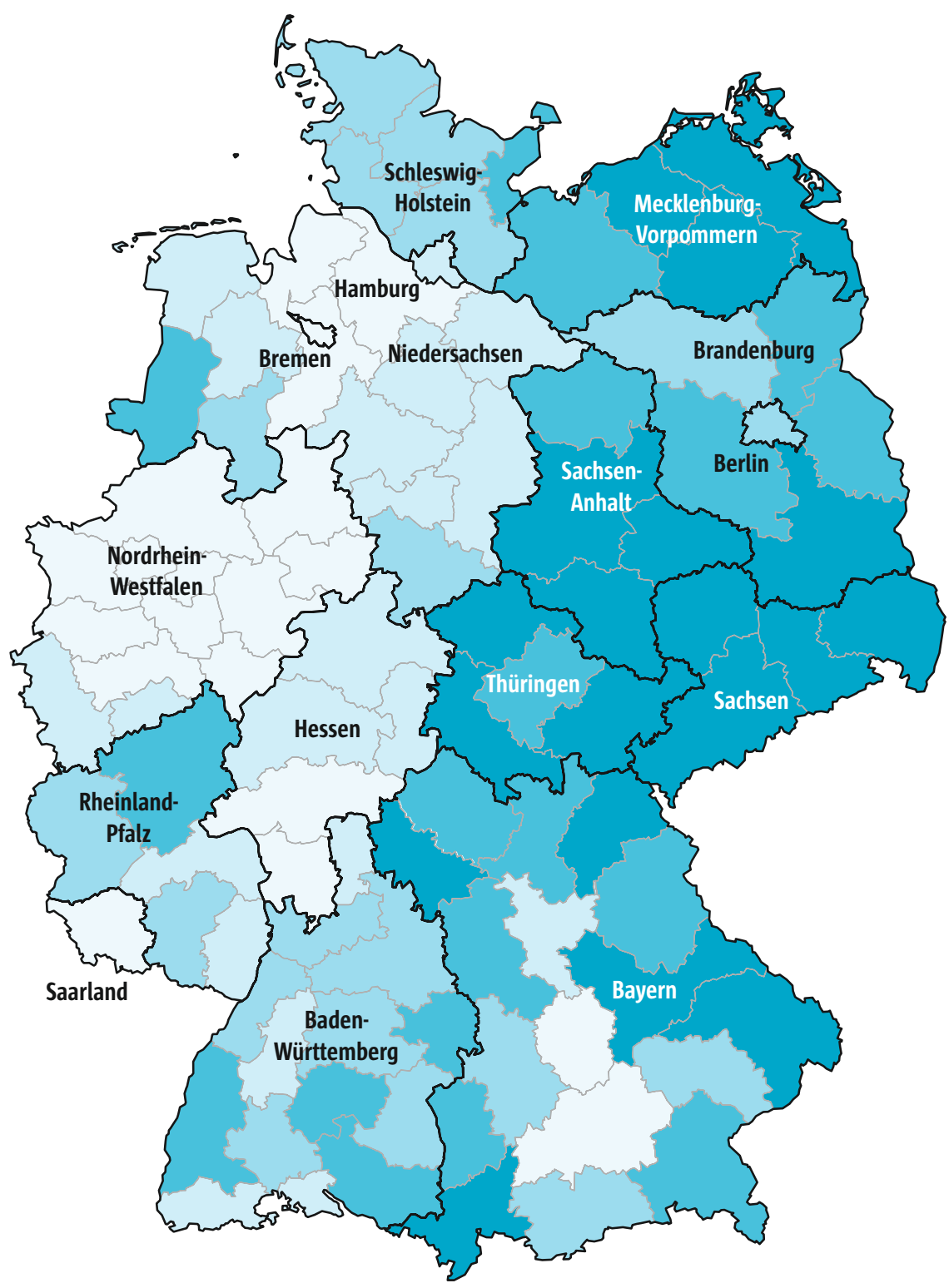

Anzahl der jährlichen Heilmittelpatienten je 100.000 Einwohner *
$\square$ 12.914-15.977
$\square$ 15.978-17.786
$17.787-18.986$
$18.987-20.488$
$20.489-26.792$

* standardisiert auf die deutsche Wohnbevölkerung

Abb. 12 Anzahl der jährlichen Heilmittelpatienten je 100.000 Einwohner nach Raumordnungsregion (2018) 


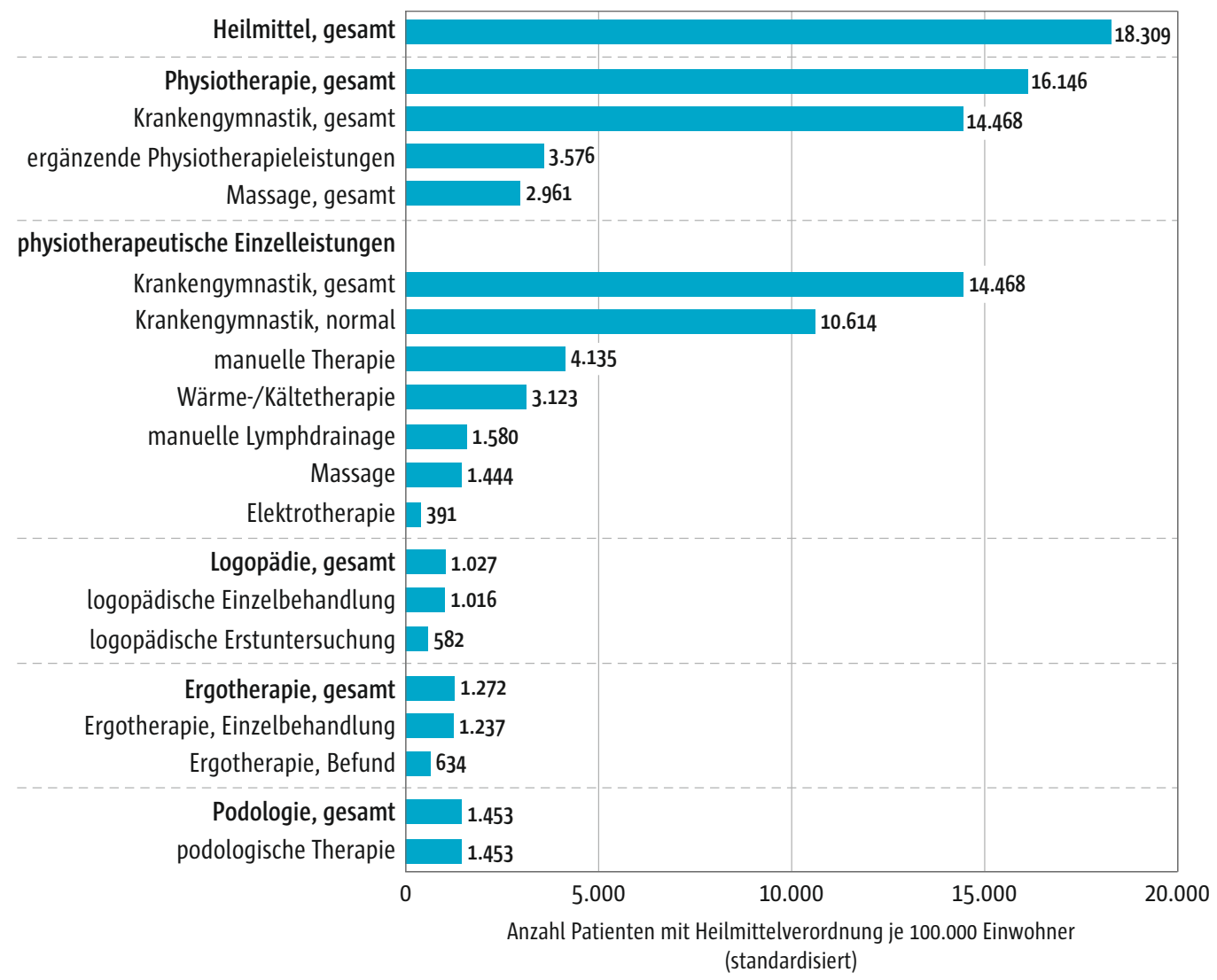

Abb. 13 Anzahl der jährlichen Patienten mit Heilmittelverordnung je 100.000 Einwohner nach Leistungsbereich und Heilmittelgruppe (2018)

bestimmten Regionen von Nord-Rhein-Westphalen (z.B. Emscher-Lippe 13.150/100.000 Einwohner) oder in Bremen (12.914/100.00o Einwohner) deutlich unter dem Bundesdurchschnitt.

Abbildung 13 zeigt die Verordnungsraten im Jahr 2018 in den vier Heilmittelbereichen Physiotherapie, Logopädie, Ergotherapie und Podologie und der darunter fallenden entsprechenden Einzelleistungen. Spitzenreiter unter den Leistungsbereichen war die Physiotherapie mit 16.146 Heilmittelpatienten je 100.00o Einwohner, die drei anderen Bereiche Logopädie, Ergotherapie und Podologie wurden mit unter 1.500 Heilmittelpatienten je 100.000 Einwohner vergleichsweise wenig in Anspruch genom- men. Innerhalb der Physiotherapie fiel gemessen an der Verordnungshäufigkeit der Krankengymnastik die größte Bedeutung zu (14.468 je 100.00o Einwohner), gefolgt von Manueller Therapie (4.135/100.0oo Einwohner) und Wärme-/Kältetherapien (3.123/100.0oo Einwohner).

Der jährliche Heilmittelbericht des WIdO bietet einen Überblick über die Entwicklung der Heilmitteleistungen im GKV-Markt. Dem Bericht nach ist seit Jahren eine kontinuierliche Zunahme der Leistungen je $1.000 \mathrm{GKV}$-Versicherte zu beobachten. Insgesamt ist die Inanspruchnahme zwischen 2007 und 2016 von 566 Heilmittelleistungen auf 628 Leistungen je $1.000 \mathrm{GKV}$-Versicherte angestiegen (Waltersbacher 2017). 


\section{Elektronischer Anhang}

Im elektronischen Anhang sind für das Jahr 2018 zusätzlich folgende Auswertungen verfügbar:

- Prävalenzen und Hospitalisierungsraten nach ICD-10-Einzeldiagnosen (erweiterte Tabelle mit mehr als hochgerechnet 1.00o Betroffenen)

- Prävalenzen und Hospitalisierungsraten auf Ebene der ICD-10-Diagnosegruppen

- die 40 häufigsten Behandlungsdiagnosen (ICD-1o-Einzeldiagnosen) bei Männern/Frauen sowie für die Altersgruppen 1 bis 17 Jahre, 18 bis 59 Jahre und 6o Jahre und älter

- die 100 häufigsten stationären Behandlungsanlässe (Hauptdiagnosen) aller voll- und teilstationären Behandlungsfälle

- Verordnungshäufigkeit aller Wirkstoffgruppen gemäß ATC-Klassifikation auf der zweiten hierarchischen Ebene (alphabetisch)

\section{Weitere:}

- Zuordnung der EBM-Facharztgruppen zu 16 Arztfachgruppen für den Versorgungs-Report

\section{Danksagung}

Besonderer Dank gilt Bettina Gerste und Dr. Dagmar Drogan, die den Teil „Daten und Analysen“ im VersorgungsReport konzeptionell aufgebaut über viele Jahre weiterentwickelt haben. Als Autorinnen der früheren Ausgaben haben sie wesentliche Teile dieses Beitrages verfasst. Ein besonderer Dank gilt auch Thomas Ruhnke für die Unterstützung bei der Datenaufbereitung.

\section{Literatur}

Bundesinstitut für Bau-, Stadt- und Raumforschung (BBSR) (2017) Raumordnungsregionen Stand 31.12.2017. URL: https://www. bbsr.bund.de/BBSR/DE/forschung/raumbeobachtung/downloads/downloadsReferenz2.html (abgerufen am 25.03.2021)

Bundesministerium für Gesundheit (2019) Gesetzliche Krankenversicherung: Mitglieder, mitversicherte Angehörige und Krankenstand. Jahresdurchschnitt 2018. (Ergebnisse der GKV-
Statistik KM1/13). Stand: 18. März 2019. URL: https://www. bundesgesundheitsministerium.de/fileadmin/Dateien/3_ Downloads/Statistiken/GKV/Mitglieder_Versicherte/KM1 ID_2018.pdf (abgerufen am 19.03.2021)

Breitkreuz J, Schüssel K, Brückner G, Schröder H (2021) Krankheitslastbestimmung mit Prävalenzen und Schweregraden auf Routinedatenbasis. Gesellschaft und Gesundheit Wissenschaft (GGW) 21:24-34

Carnarius S, Heuer I, Stausberg I (2018) Diagnosis Coding in German Medical Practices: A Retrospective Study Using Routine Data. Gesundheitswesen 80:1000-1005

DIMDI (2018a) Internationale statistische Klassifikation der Krankheiten und verwandter Gesundheitsprobleme, 10. Revision German Modification Version 2018. URL: https://www.dimdi. de/static/de/klassifikationen/icd/icd-10-gm/kode-suche/ htmlgm2018/ (abgerufen am 19.03.2021)

DIMDI (2018b) Anatomisch-therapeutisch-chemische Klassifikation mit Tagesdosen. Amtliche Fassung des ATC-Index mit DDD-Angaben für die Bundesrepublik Deutschland im Jahre 2018. URL: https://www.dimdi.de/dynamic/.downloads/arzneimittel/ atcddd/atc-ddd-amtlich-2018.pdf (abgerufen am 19.03.2021)

Fricke U, Günther |, Niepraschk-von-Dollen K, Zawinell A (2018) Anatomisch-therapeutisch-chemische Klassifikation mit Tagesdosen für den deutschen Arzneimittelmarkt. ATC-Index mit DDDAngaben. URL: https://www.wido.de/fileadmin/Dateien/Dokumente/Publikationen_Produkte/Arzneimittel-Klassifikation/ wido_arz_atc_gkv-ai_2018.pdf (abgerufen am 19.03.2021)

Gerste B, Drogan D, Günster C (2016) Diagnosehäufigkeit und Inanspruchnahme von Gesundheitsleistungen. In: Klauber |, Günster C, Gerste B, Robra B-P, Schmacke N (Hrsg.) Versorgungs-Report 2015/2016. Schattauer GmbH, Stuttgart, S. 391-445

Gerste B, Günster C (2014) Diagnosehäufigkeit und Inanspruchnahme von Gesundheitsleistungen. In: Klauber I, Günster C, Gerste B, Robra BP, Schmacke N (Hrsg.) Versorgungs-Report 2013/2014. Schwerpunkt: Depression. Schattauer, Stuttgart, S. $258-308$

Gerste B, Günster C (2012) Erkrankungshäufigkeiten und Inanspruchnahme von Gesundheitsleistungen. In: Günster C, Klose I, Schmacke N (Hrsg.) Versorgungs-Report 2012. Schwerpunkt: Gesundheit im Alter. Schattauer, Stuttgart/New York, S. 313-384

Hartmann I, Weidmann C, Biehle R (2016) Validierung von GKVRoutinedaten am Beispiel von geschlechtsspezifischen Diagnosen. Gesundheitswesen 78:e53-e58

Hoffmann F, Icks A (2012) Unterschiede in der Versichertenstruktur von Krankenkassen und deren Auswirkungen für die Versorgungsforschung: Ergebnisse des Bertelsmann Gesundheitsmonitors. Das Gesundheitswesen 74:291-297. doi:10.1055/ s-0031-1275711

Jaunzeme I, Eberhard S, Geyer S (2013) Wie „repräsentativ“ sind GKV-Daten? Demografische und soziale Unterschiede und Ähnlichkeiten zwischen einer GKV-Versichertenpopulation, der Bevölkerung Niedersachsens sowie der Bundesrepublik am Beispiel der AOK Niedersachsen. Bundesgesundheitsblatt, Gesundheitsforschung, Gesundheitsschutz 56:447-454. doi:10.1007/s00103-012-1626-9 
Kreienbrock L, Pigeot I, Ahrens W (2012) Epidemiologische Methoden. In: Epidemiologische Methoden, 5. Auflage. Springer-Verlag, Berlin Heidelberg

Schmuker C, Beydoun G, Günster C (2019) Diagnosehäufigkeit und Inanspruchnahme von Gesundheitsleistungen. In: Günster $C$, Klauber I, Robra B-P, Schmacke N, Schmuker C (Hrsg.) Versorgungs-Report Früherkennung. Medizinisch Wissenschaftliche Verlagsgesellschaft, Berlin, S. 211-248

Schubert I, Ihle P, Koster I (2010) Interne Validierung von Diagnosen in GKV-Routinedaten: Konzeption mit Beispielen und Falldefinition. Das Gesundheitswesen 72:316-322

Schwabe U, Ludwig W-U, Paffrath D, Klauber I (2018) Arzneiverordnungen 2018 im Überblick. In: Schwabe U, Paffrath D, Ludwig W-D, Klauber I (Hrsg.) Arzneiverordnungs-Report 2019. Springer, Berlin/Heidelberg, S. 4-30

Statistisches Bundesamt (2016) Gesundheit - Fallpauschalenbezogene Krankenhausstatistik (DRG-Statistik). Diagnosen, Prozeduren, Fallpauschalen und Case Mix der vollstationären Patientinnen und Patienten in Krankenhäusern 2016. URL: https://www.destatis.de/DE/Themen/GesellschaftUmwelt/ Gesundheit/Krankenhaeuser/Publikationen/Downloads-Krankenhaeuser/fallpauschalen-krankenhaus-2120640167004. pdf; jsessionid=D8E623A6E05D2146F69BBCDFBCE22FB6. live731?__blob=publicationFile (abgerufen am 23.03.2021)

Statistisches Bundesamt (2021a) Bevölkerung: Deutschland, Stichtag. URL: https://www-genesis.destatis.de/genesis/online (abgerufen am 19.03.2021)

Statistisches Bundesamt (2021b) Anzahl der abgerechneten Fallpauschalen der vollstationären Patientinnen und Patienten in Krankenhäusern. Die Tabelle wurde am 19.03.2021 unter www.gbe-bund.de erstellt.

Tillmanns H, Schillinger G, Dräther H (2019) Früherkennung bei Erwachsenen in der gesetzlichen Krankenversicherung: Ergebnisse einer AOK-Sekundärdatenanalyse. In: Günster C, Klauber I, Robra BP, Schmacke N, Schmuker C (Hrsg.) Versorgungs-Report Früherkennung. Medizinisch Wissenschaftliche Verlagsgesellschaft, Berlin

Waltersbacher A (2017) Heilmittelbericht 2017. URL: https://www. wido.de/fileadmin/Dateien/Dokumente/Publikationen_Produkte/Buchreihen/Heilmittelbericht/wido_hei_hmb_2017. pdf (abgerufen am 26.03.2021)

Zok K (2015) Private Zusatzleistungen in der Arztpraxis. Ergebnisse einer bundesweiten Repräsentativ-Umfrage unter gesetzlich Versicherten. WIdOmonitor 12 

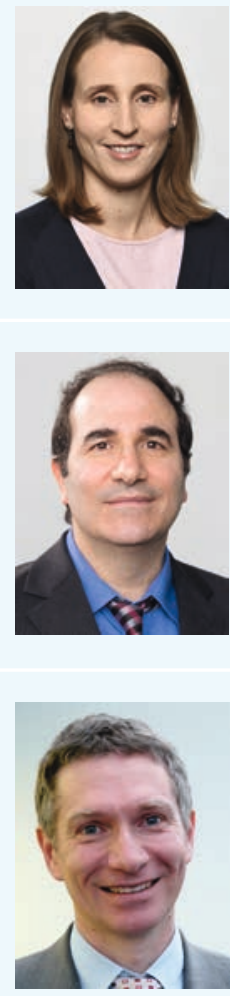

\section{Caroline Schmuker}

Studium der Volkswirtschaftslehre an der Universität Heidelberg. Weiterqualifikation im Fachbereich Epidemiologie an der London School of Hygiene and Tropical Medicine (LSHTM). Berufliche Stationen: 2009 bis 2011 Trainee am Wissenschaftlichen Institut der AOK (WIdO) im Bereich Gesundheitspolitik und Systemanalysen, zwischen 2012 und 2017 wissenschaftliche Mitarbeiterin am IGES Institut Berlin. Seit November 2017 wissenschaftliche Mitarbeiterin im Bereich Qualitäts- und Versorgungsforschung am WId0.

\section{Ghassan Beydoun}

Studium der Informatik in Berlin. Langjährige Berufserfahrung in der Software- und Datenbankentwicklung im Gesundheitswesen. Seit 2012 Mitarbeiter des Wissenschaftlichen Instituts der AOK (WIdO) im Bereich Qualitäts- und Versorgungsforschung, in verschiedenen Projekten tätig.

\section{Dipl.-Math. Christian Günster}

Studium der Mathematik und Philosophie in Bonn. Seit 1990 beim Wissenschaftlichen Institut der AOK (WId0). Von 2002 bis 2008 Mitglied des Sachverständigenrates nach $\oint 17 \mathrm{~b}$ KHG des Bundesministeriums für Gesundheit. Leitung des Bereichs Qualitäts- und Versorgungsforschung. Mitherausgeber des Versorgungs-Reports. Arbeitsschwerpunkte sind Methoden der Qualitätsmessung und Versorgungsanalysen mittels Routinedaten. 